\title{
Myostatin is a negative regulator of adult neurogenesis in zebrafish
}

\#Vishnu Muraleedharan Saraswathy ${ }^{1,2}$, \#Lili Zhou1,2, Brooke Burris ${ }^{1,2}$, Deepika Dogra ${ }^{3,4}$, Sven Reischauer ${ }^{3,5,6}$, and Mayssa H. Mokalled ${ }^{1,2,7}$

\#Co-authors

${ }^{1}$ Department of Developmental Biology

Washington University School of Medicine, St. Louis, MO, USA 63110.

${ }^{2}$ Center of Regenerative Medicine

Washington University School of Medicine, St. Louis, MO, USA 63110.

${ }^{3}$ Department of Developmental Genetics, Max Planck Institute for Heart and Lung Research, Bad Nauheim 61231, Germany

${ }^{4}$ Department of Medical Genetics, Cumming School of Medicine, University of Calgary, Calgary, AB T2N 4N1, Canada

${ }^{5}$ Medical Clinic I, (Cardiology/Angiology) and Campus Kerckhoff, Justus-Liebig University, Giessen, 35392 Giessen, Germany.

${ }^{6}$ The Cardio-Pulmonary Institute, Frankfurt, Germany.

${ }^{7}$ Author for correspondence: mmokalled@wustl.edu 


\section{ABSTRACT}

1 Intrinsic and extrinsic inhibition of axonal and neuronal regeneration obstruct spinal cord (SC)

2 repair in mammals. In contrast, adult zebrafish achieve functional recovery after SC damage.

3 While studies of innate SC regeneration have focused on axon regrowth as a primary repair 4 mechanism, how local neurogenesis impacts functional recovery is unknown. We uncovered 5 dynamic expression of myostatin $b$ (mstnb) in a niche of dorsal ependymal progenitors after 6 complete SC transection in zebrafish. Genetic loss-of-function in mstnb impaired functional 7 recovery, although glial and axonal bridging across the lesion were unaffected. Using a series of 8 transgenic reporter lines, we quantified the numbers of stem, progenitor, and neuronal cells in the 9 absence of mstnb. We found neural stem cell proliferation was reduced, while newborn neurons 10 were increased in mstnb null tissues, suggesting mstnb is a negative regulator of neurogenesis.

11 Molecularly, neuron differentiation genes were upregulated, while the neural stem cell

12 maintenance gene fgf1b was downregulated in mstnb mutants. Finally, we show that human

13 FGF1 treatment rescued neuronal gene expression in mstnb mutants. These studies uncover 14 unanticipated neurogenic functions for mstnb in adult zebrafish, and establish the importance of local neurogenesis for functional SC repair. 


\section{INTRODUCTION}

16 Traumatic spinal cord injury (SCl) causes irreversible neuronal and systemic deficits (Hachem et 17 al., 2017; Silva et al., 2014; Singh et al., 2014). In mammals, axon regrowth and neurogenesis 18 are impeded by intrinsic and extrinsic inhibitory mechanisms that obstruct spinal cord (SC) 19 regeneration (Alizadeh et al., 2019; Oyinbo, 2011; Sofroniew, 2018; Tran et al., 2021). Although 20 multiple cell types including astrocytes and oligodendrocyte progenitor cells proliferate after $\mathrm{SCl}$, 21 the mammalian SC is incapable of generating mature neurons in vivo (Horky et al., 2006; Horner 22 et al., 2000; Yamamoto et al., 2001). In contrast with mammals, highly regenerative vertebrates 23 including teleost fish spontaneously recover after SCl. Following complete transection of SC 24 tissues, adult zebrafish extend glial and axonal bridges across the lesion and achieve functional 25 recovery within 6 to 8 weeks of injury. In addition to axon regrowth from hindbrain neurons, 26 zebrafish regenerate motor neurons and interneurons around the lesion site (Becker et al., 1998; 27 Becker et al., 1997; Kuscha et al., 2012a; Mokalled et al., 2016; Reimer et al., 2008). Yet, the contribution of local neurogenesis to functional recovery and the mechanisms that coordinate the regeneration of different neuronal subtypes remain to be determined.

Ependymal radial glial cells (ERGs) line the brain ventricles and SC central canal. ERGs coexpress astroglial (gfap and blbp) and progenitor (sox2 and hey) cell markers, and comprise populations of neurogenic stem cells in adult zebrafish (Kroehne et al., 2011; März et al., 2010; Ogai et al., 2014; Reimer et al., 2009; Than-Trong et al., 2020; Than-Trong et al., 2018). In uninjured neural tissues, the majority of ERGs are non-dividing, quiescent cells that possess epithelial-like features (Barbosa et al., 2015; Chapouton et al., 2010; März et al., 2010). Following brain and SC damage, ERGs undergo widespread proliferation and are thought to act as a major source of regenerated neurons (Adolf et al., 2006; Barbosa et al., 2015; Grandel et al., 2006; Kroehne et al., 2011). Activated ERGs undergo 3 modes of cell division: 1) symmetric division into ERGs, 2) asymmetric division into ERG and neural progenitors, or 3) symmetric division to generate differentiated neurons (Barbosa et al., 2015; Rothenaigner et al., 2011). Distinct lineagerestricted ERG domains emerge during SC regeneration in zebrafish. ERGs within the progenitor motor neuron (pMN) domain express olig2 and generate is/1/2 and $h b 9^{+}$motor neurons after $\mathrm{SCl}$ (Reimer et al., 2008). On the dorsal and ventral sides of the pMN, ERGs give rise to $v s \times 1^{+} \mathrm{V} 2$ interneurons and serotonergic neurons, respectively (Barreiro-Iglesias et al., 2015; Kuscha et al.,

45 2012b). We recently showed that ventral ERGs undergo epithelial-to-mesenchymal transition 46 (EMT), and that EMT is required for glial bridging and functional regeneration (Klatt Shaw et al., 
2021). Thus, despite their morphological similarities, ERGs elicit compartmentalized injury responses and proliferate into lineage-restricted progenitors during SC regeneration.

Myostatin (Mstn), also known as Growth differentiation factor 8 (Gdf8), is a Tgf- $\beta$ superfamily member. Upon binding Activin type 2 and type 1 receptors, Mstn induces the phosphorylation and nuclear translocation of Smad 2/3 to regulate target gene expression (Massagué, 2012; Sartori et al., 2014; Sharma et al., 2015). Spontaneous and targeted Mstn loss-of-function mutations lead to double muscle phenotypes in zebrafish, mice, cattle and humans (Dogra et al., 2017;

54 Kambadur et al., 1997; Schuelke et al., 2004; Whittemore et al., 2003). Mechanistically, Mstn controls lineage progression within myogenic stem cells (satellite cells) and progenitor cells (myoblasts). During muscle development, Mstn inhibits myoblast differentiation via negative regulation of the myogenic transcription factors MyoD and Myogenin. Mstn also suppresses satellite cell proliferation, differentiation, and muscle regeneration (Langley et al., 2002; McCroskery et al., 2003; McCroskery et al., 2005). However, it remains unclear whether recombinant MSTN proteins inhibit or stimulate myoblast proliferation (Rodgers et al., 2014; Taylor et al., 2001). Equally conflicting effects were reported for recombinant MSTN proteins on neuronal proliferation and neurite outgrowth in vitro (Kerrison et al., 2005; Wu et al., 2003), suggesting Mstn functions are dose- and context-dependent and that in vivo studies are required to decipher the role of Mstn in the nervous system.

Tgf- $\beta$ signaling directs immune, fibrotic, or regenerative injury responses across tissues and species. As zebrafish SCs regenerate without fibrotic scarring, we postulated that Tgf- $\beta$ signaling is pro-regenerative in adult zebrafish. By surveying the expression of Tgf- $\beta$ ligands after $\mathrm{SCl}$, we found mstnb is induced in dorsal ERGs of lesioned SC tissues. mstnb mutants showed normal baseline swim capacity but failed to achieve functional recovery following $\mathrm{SCl}$, despite having normal axonal and glial bridging across the lesion. Mstnb inhibition using genetic loss-of-function and pharmacological approaches enhanced neurogenesis and diminished ERG proliferation. A series of transgenic reporter lines was used to quantify the numbers of neural stem cells (NSCs), intermediate neural progenitors (iNPs), and interneurons. These studies revealed NSC proliferation was reduced, while regenerating neurons were increased in mstnb mutants. RNA sequencing showed neuron differentiation genes were upregulated in mstnb mutants. Finally, we show that the neural stem cell maintenance gene fgf1b was downregulated in mstnb mutants, and that human FGF1 treatment rescued neuronal gene expression. These studies indicate that 
induced mstnb expression is required to maintain the potency and self-renewal of neurogenic ERGs during SC regeneration.

\section{RESULTS}

81 Tgf- $\beta$ signaling is activated in dorsal ependymal progenitors after $\mathbf{S C I}$.

82 Injuries to the central and peripheral nervous systems induce Tgf- $\beta$ signaling across vertebrates.

83 In mammals, Tgf- $\beta$ activation directs a range of regenerative and anti-regenerative cell responses

84 including immune cell activation, neurite outgrowth, and scar formation (Li et al., 2017). In

85 zebrafish larvae, the anti-inflammatory effects of Tgfb1a are required for SC regeneration

86 (Keatinge et al., 2021). We postulated that Tgf- $\beta$ signaling is pro-regenerative in adult zebrafish.

87 To explore this hypothesis, we first surveyed Smad3 phosphorylation as a readout of Tgf- $\beta$ activity 88 after zebrafish SCI. By immunohistochemistry, phosphorylated Smad3 (pSmad3) was strongly induced in dorsal SC tissues at 1 week post-injury (wpi) (Fig. 1A). pSmad3 expression gradually diminished between 2 and 3 wpi, and was minimally expressed in uninjured SCs (Fig. 1A). At 1 wpi, pSmad $3^{+}$cells accounted for $7 \%$ of dorsal SC cells, and were reduced by 3 wpi relative to 1 wpi (Fig 1B, S1A). To determine the identity of Tgf- $\beta$ responsive cells after $\mathrm{SCl}$, we co-labelled pSmad3 with either the neuronal markers $\mathrm{HuC}$ and $\mathrm{HuD}(\mathrm{HuC} / \mathrm{D})$ or the ependymal progenitor marker Sox2. At $1 \mathrm{wpi}$, we rarely observed vesicular pSmad3 expression in some $\mathrm{HuC} / \mathrm{D}^{+}$ neurons. However, the majority of $\mathrm{HuClD}^{+}$neurons were pSmad3- (Fig. S1B). pSmad3 was primarily expressed at high levels in Sox $2^{+}$ERGs (Fig. 1C). Quantification revealed 66 to $76 \%$ of pSmad $3^{+}$cells were Sox $2^{+}$across timepoints (Fig. 1D, S1C). By EdU incorporation, 10\% of pSmad $3^{+}$cells were proliferative at 1 wpi in dorsal SCs, and $\mathrm{pSmad} 3^{+}$cell proliferation decreased to baseline levels by 3 wpi (Fig. S1D, E). These findings indicated Tgf- $\beta$ signaling is activated in dorsal ERGs after SCI.

\section{mstnb expression is upregulated in dorsal ependymal progenitors after SCI.}

102 To explore mechanisms of Tgf- $\beta$ activation during SC regeneration, we used a previously 103 published RNA-seq dataset to survey the expression of Tgf- $\beta$ ligands at 2 wpi (Mokalled et al., 104 2016). We found $m s t n b, b m p 2 a / b$, and $b m p 5$ are upregulated, while gdf3, gdf6a, gdf9, bmp6, and $105 n d r 1$ are downregulated after injury relative to uninjured SC tissues (Fig. S1F). By fluorescence 106 in situ hybridization, mstnb expression was induced in dorsal SC tissues between 1 and 2 wpi 107 and decreased at 3 wpi (Fig. 1E-G). mstnb transcripts were not detectable in uninjured SCs (Fig. 108 1E-G). Co-labeling of mstnb transcripts with either ependymal Sox2 or neuronal HuC/D showed 109 mstnb expression restricted to a subset of Sox $2^{+}$ERGs in dorsal SCs (Fig. 1F). mstnb transcripts 
110 were excluded from neuronal cell bodies (Fig. 1G). These studies revealed mstnb expression is 111 induced in a subset of dorsal ERGs after $\mathrm{SCl}$, and suggested mstnb expression correlates with

112 Tgf- $\beta$ activation in dorsal SC tissues during SC regeneration.

\section{3 mstnb is required for functional SC repair.}

114 To examine the role of mstnb during SC regeneration, we analyzed the extent of functional and 115 cellular recovery in genetic zebrafish mutants (mstnb ${ }^{\text {bns5 }}$ ) (Dogra et al., 2017) (Fig. 2A). mstnb 116 mutants are adult viable, and elicit skeletal and cardiac muscle hyperplasia. Interestingly, the 117 growth phenotypes associated with mstnb mutants are thought to be muscle specific (Dogra et 118 al., 2017). To establish baseline motor function, we first assessed the swim capacities of wild119 type, mstnb heterozygous $\left(m s t n b^{+/}\right)$and homozygous $\left(m s t n b^{-/}\right)$siblings in an enclosed swim 120 tunnel under increasing water current velocities (Klatt Shaw and Mokalled, 2021; Klatt Shaw et 121 al., 2021; Mokalled et al., 2016) (Fig. 2B). In this swim endurance assay, wild-type animals swam 122 for 41 min before reaching exhaustion. $m s t n b^{+/}$and $m s t n b^{-/}$fish showed comparable swim 123 functions, averaging 43 and $39 \mathrm{~min}$ of swim time, respectively. These results indicated $m s t n b$ 124 mutants show normal swim capacity and suggested the muscle phenotype of mstnb mutants does 125 not impact swim endurance. Next, we performed SC transections on $\mathrm{mstn}^{-/-}$and control siblings 126 and evaluated their functional regeneration between 2 and 6 wpi. $m s t n b^{+/}$fish displayed normal 127 swim capacity at 2 and 4 wpi, but their functional recovery was slightly compromised at 6 wpi (Fig. $1282 \mathrm{C}$ ). Relative to wild-type controls, functional recovery was $50 \%$ reduced in $m s t n b^{-/}$fish at 2,4 , 129 and 6 wpi (Fig. 2C).

To further rule out the contribution of skeletal muscle overgrowth to the functional regeneration 131 output of mstnb mutants, we tracked the swim behavior of mstnb mutants in the absence of water 132 current or under a constant, low current velocity of $10 \mathrm{~cm} / \mathrm{sec}$ (Fig. 2D). We reasoned that, unlike 133 the endurance test that required fish to swim against increasing current velocities, swim behavior 134 under minimal current velocity is less likely to be dependent on muscle function. Fish position in 135 the swim tunnel ( $Y$ position), percent activity, and burst frequency were quantified to assess 136 overall swim competence. In this assay, $m s t n b^{-/}$animals stalled in the back quadrant of the swim 137 tunnel (Fig. 2E), were 65\% less active than their wild-type siblings (Fig. 2F), and displayed less 138 bursts under low current velocity (Fig. 2G). Consistent with a partial regeneration phenotype in 139 heterozygous fish, mstnb $^{+/-}$fish were $40 \%$ less active and their burst frequency was reduced by $14035 \%$ relative to wild types at 2 wpi. Swim parameters were comparable between $m s t n b^{+/}$and 141 wild-type siblings at 4 and 6 wpi, and were not statistically significant at 2 wpi (Fig. 2E-G). 
To date, cellular growth across the lesion site has served as a primary readout of cellular regeneration in zebrafish (Goldshmit et al., 2012; Mokalled et al., 2016; Reimer et al., 2013). Glial

144 bridging and axon tracing assays were performed to evaluate the extents of glial and axonal 145 regeneration across the lesion site (Fig. 2A). By Gfap immunostaining, glial bridging was 146 unaffected in $\mathrm{mstnh}^{-/}$animals compared to wild-type siblings at 2 wpi (Fig. $2 \mathrm{H}$ ). At 4 wpi, 147 anterograde axon tracing using Biocytin showed comparable axon regrowth in proximal and distal 148 SC sections between $m s t n b^{-/}$and control animals (Fig. 2l). Together, these studies indicated 149 mstnb is required for functional SC repair but is dispensable for glial bridging and axonal regrowth 150 across the lesion. These findings prompted us to investigate mechanisms of SC regeneration that 151 are independent of glial bridging and axon growth.

\section{mstnb is a negative regulator of adult neurogenesis after $\mathrm{SCl}$}

153 In addition to glial bridging and axon regrowth, zebrafish regenerate lost motor neurons and 154 interneurons around the lesion site (Barreiro-Iglesias et al., 2015; Kuscha et al., 2012b; Reimer et al., 2008). Dorsal ependymal progenitors are thought to give rise to regenerating interneurons in dorsal SC tissues after injury. Since mstnb is expressed in dorsal ERGs after SCl and mstnb mutants did not show glial bridging or axon growth defects, we postulated that $m s t n b$ plays a role in adult neurogenesis in zebrafish and that local neurogenesis around the lesion is required for functional SC repair. To test this hypothesis, we first examined the proliferation rates of Sox $2^{+}$

160 ERGs and of regenerating HuC/D+ neurons in mstnb mutants. Uninjured and injured $m s t n b^{-/-}$and 161 wild-type siblings were subjected to SCl and to a single EdU pulse 24 hours prior to SC collection 162 and histological analysis (Fig. 3A and S2A). Cell counts revealed a significant increase in HuC/D $163 \mathrm{EdU}^{+}$neurons in dorsal SCs with 1 wpi SCs showing the most pronounced differences (Fig. 3B, $164 \mathrm{C}$ and S2B). At $1 \mathrm{wpi}, 7.7 \%$ of $\mathrm{HuC}^{+} \mathrm{D}^{+}$neurons were $\mathrm{EdU}^{+}$in wild-type SCs (Fig. S2B), and 165 accounted for $0.9 \%$ of dorsal SC cells (Fig. 3C). Conversely, $15.2 \%$ of $\mathrm{HuC}^{+} \mathrm{D}^{+}$neurons were $166 \mathrm{EdU}^{+}$in $\mathrm{mstnh}^{-/}$SCs (Fig. S2B), accounting for $1.5 \%$ of dorsal SC cells (Fig. 3C). The rates of 167 neurogenesis where attenuated in wild-type SCs at 2 and 3 wpi relative to 1 wpi. However, $m s \operatorname{tn} b$ 168 mutants showed increased neurogenesis at 3 wpi (Fig. 3C and S2B). These differences were 169 blunted in cell counts from total SCs (Fig. S2C), suggesting neuronal differentiation is specifically 170 increased in dorsal SC tissues of mstnb mutants. On the other hand, we observed a minor, non171 significant decrease in the number of Sox2 ${ }^{+} \mathrm{EdU}^{+}$ERGs in $m s t n b^{-/}$SCs at 1 and 3 wpi (Fig. 3D, $172 \mathrm{E}$ and S2D, E). These findings indicated the rates of neurogenesis are increased in mstnb 173 mutants. 
To evaluate how snapshots of increased neurogenesis at 1 wpi could impact the numbers of regenerating neurons in mstnb mutants, we performed SC transections on stnh $^{-/}$and wild-type fish followed by daily EdU injections for 1 or 2 wpi (Fig. 4A and S3A). In this assay, daily EdU labeling allowed us to estimate the total numbers of regenerating neurons $\left(\mathrm{HuC} \mathrm{D}^{+} \mathrm{EdU} \mathrm{U}^{+}\right.$ neurons), and the extent of self-renewal in ependymal progenitors (Sox2 ${ }^{+} \mathrm{EdU}^{+} \mathrm{ERGs}$ ). Considering the dynamic rates of neurogenesis along the rostro-caudal axis, we quantified cell numbers at 150, 450, and $750 \mu \mathrm{m}$ rostral to the lesion. At 1 wpi and in wild-type sections proximal

181 to the lesion site $(-150 \mu \mathrm{m}), 10.9 \%$ of dorsal SC cells were HuC/D+ neurons (Fig. $3 \mathrm{C}$ ), and $2.7 \%$ 182 of dorsal SC cells were regenerating neurons $\left(\mathrm{HuC}^{+} \mathrm{EdU}^{+}\right)(\mathrm{Fig} .3 \mathrm{D})$. On the other hand, 19\% 183 of dorsal SC cells were HuC/ $\mathrm{D}^{+}$neurons (Fig. 3C), and $4.3 \%$ of dorsal SC cells were regenerating 184 neurons $\left(\mathrm{HuC} / \mathrm{D}^{+} \mathrm{EdU}^{+}\right.$) in $\mathrm{mstnh}^{-/-} \mathrm{SCs}$ (Fig. 3D). Quantifications from total SC tissues confirmed 185 that the increase in regenerating neurons in mstnb mutants is specific to dorsal SCs and blunted 186 in total SCs (Fig. S3B,C). At 2 wpi, the numbers of HuC/D+ EdU neurons continued to be elevated 187 in mstnb $^{-/}$SCs (Fig. 4D). Intriguingly, the overall increase in the total numbers of neurons was 188 less pronounced at this time point (Fig. 4C), suggesting mstnb mutants elicit an early wave of 189 increased neurogenesis at $1 \mathrm{wpi}$, but that compensatory mechanisms may be activated at later 190 time points to counteract the loss of mstnb. Finally, despite increased neurogenesis rates in $191 \mathrm{mstnh}^{-/}$fish, the numbers of Sox $2^{+}$and Sox ${ }^{+} \mathrm{EdU}^{+}$ERGs were comparable across genotypes 192 (Fig. 4F,G), suggesting ERG self-renewal was maintained at normal levels in mstnb mutants. 193 These studies revealed a 2-fold increase in regenerating neurons in mstnb mutants, and are 194 consistent with mstnb acting as a negative regulator of adult neurogenesis in zebrafish.

Human MSTN proteins are translated as inactive full-length precursors that undergo proteolytic processing into mature MSTN peptide and MSTN proform (pro-MSTN) peptide. proMSTN exhibits high binding affinity for Myostatin and inhibits its function (Zhu et al., 2000). To examine whether the global effects of mstnb mutants could be reproduced by local Mstnb inhibition, we injured wild-type animals and performed daily injections of human recombinant MSTN Proform (pro-MSTN) peptide adjacent to the lesion site (Fig. S4A). We then assessed the numbers of $\mathrm{HuC} / \mathrm{D}^{+}$neurons and Sox2 ${ }^{+} \mathrm{ERGs}$ at $1 \mathrm{wpi}$, corresponding to 6 days after initial treatment. We found $\mathrm{HuC} / \mathrm{D}^{+}$neurons were increased by $12 \%$ upon pro-MSTN treatment, though 203 these differences were not significant (Fig. S4B). On the other hand, the numbers of Sox2 204 progenitors were decreased by $20 \%$ in pro-MSTN injected fish relative to vehicle controls (Fig. 205 S4C). Consistent with genetic loss-of-function of mstnb, pharmacological Mstn inhibition at the 
lesion site disrupted the relative ratios of $\mathrm{HuC} / \mathrm{D}^{+}$neurons and Sox2 ${ }^{+} \mathrm{ERG}$ towards increased neurogenesis.

mstnb mutants exhibit increased neuronal differentiation after $\mathrm{SCl}$

209 Our proliferation assays showed increased neurogenesis and suggested the rate of ERG self210 renewal was slightly decreased in the absence of mstnb. To dissect the cellular basis for this 211 phenotype, we evaluated the numbers of neural stem cells (NSCs) and intermediate neural 212 progenitors (iNPs) in mstnb mutants at baseline, 1 and 2 wpi (Fig. 5A). We first combined a nestin 213 reporter transgene (nes:GFP) with $m s t n b^{-/}$background to quantify NSCs in nes:GFP; $m s t n b^{-/}$fish 214 (Lam et al., 2009) (Fig. 5B-D and S5A-C). nes:GFP ${ }^{+}$NSCs were rarely identified in uninjured SC sections from either mutant or wild-type animals, but were readily detectable after $\mathrm{SCl}$ (Fig. 5C and S5A). The proportions of nes:GFP ${ }^{+}$cells in dorsal or total SC tissues were comparable between $m s t n b^{-/-}$and wild-type siblings at either 1 or 2 wpi (Fig. 5C and S5A). At these time points, NSC proliferation showed decreasing trends in mstnb mutants (Fig. 5D), and was statistically significant when the numbers of nes:GFP ${ }^{+} \mathrm{PCNA}^{+}$cells were normalized to nes:GFP ${ }^{+} \mathrm{NSCs}_{\text {(Fig. }}$ S5C). NSC proliferation was less pronounced in quantifications from total SC sections (Fig. S5B). These findings revealed NSC proliferation is reduced in mstnb mutants at $1 \mathrm{wpi}$.

The number of nes:GFP ${ }^{+}$NSCs were unaltered in mstnb mutants despite their decreased proliferation rate. We postulated that mstnb loss-of-function may bias NSC fate towards neuronal differentiation, and that compensatory mechanisms upstream of NSC activation maintain their total numbers across genotypes. To test this hypothesis, we examined the number of iNPs using $d b \times 1 b$ :GFP transgene bred into a mstnb $^{-/}$background (Pierani et al., 2001; Satou et al., 2012) (Fig. 5E-G and S5D,E). The proportion of $d b \times 1 b:$ GFP $^{+}$iNPs averaged between 2.9 and $4.3 \%$ of dorsal SC cells across time points, and showed an elevated trend in mstnh $^{-/}$relative to wild-type siblings at 2 wpi (Fig. 5F and S5D). These phenotypes were more pronounced in quantifications

230 of $d b \times 1 b: G^{2} P^{+}$PCNA $^{+}$iNPs. Relative to wild-type siblings, $m s t n b^{-/}$iNP proliferation was minimal 231 prior to injury, increased by 2.3-fold at 1 wpi, and was normalized to wild-type levels by 2 wpi 232 (Fig. 5G and S5E). These findings are consistent with accelerated neurogenesis in mstnb 233 mutants.

234 To examine the differentiation and relative distribution of iNP-derived neurons after $\mathrm{SCl}$, we 235 labeled glycinergic neurons in msntb mutants using slc6a5:GFP reporter line (Fig. 5H-J and S5F) 236 (McLean et al., 2007). At 1 wpi, the proportions of glycinergic neurons comprised 3.9\% and 3.4\% 
237 of dorsal SC cells in $m s t n b^{-/}$and wild-type fish, respectively (Fig. 5I and S5F). By 2 wpi, glycinergic

238 neurons accounted for $1.2 \%$ of dorsal SC cells in wild-type controls, but increased to $2.7 \%$ of 239 dorsal cells in $m s t n b^{-/-}$SCs (Fig. 5I and S5F). By quantifying the proportions of glycinergic neurons 240 within $\mathrm{HuC} / \mathrm{D}^{+}$neurons, glycinergic neurons accounted for $6.1 \%$ and $3.7 \%$ of neurons in wild-type 241 SC tissues at 1 and 2 wpi, respectively (Fig. 5J). These proportions were 2-fold elevated in $m s t n b$ 242 mutants, accounting for $12 \%$ of neurons at 1 wpi and $8.5 \%$ of neurons at 2 wpi (Fig. 5J). Together, 243 these results indicated mstnb mutants exhibit an expansion in glycinergic neurons, which are 244 overrepresented relative to other neuronal cell populations.

\section{Neuronal genes are upregulated in mstnb mutants}

246 To determine the molecular mechanisms by which mstnb regulates the rates of neurogenesis, we deep sequenced SC tissues from $\mathrm{mstnh}^{-/}$and wild-type siblings at $1 \mathrm{wpi}$, as well as uninjured controls (Fig. 6A-C). Principle component analysis confirmed clustering of biological replicates, and highlighted four distinct molecular signatures that are both injury- and genotype-induced (Fig. 6A). At 1 wpi, 61 genes were downregulated and 359 genes were upregulated in $\mathrm{mstnh}^{-/} \mathrm{SCs}$, suggesting mstnb may be a negative regulator of gene expression after SCI (Fig. 6C). Genes upregulated in $\mathrm{mstnh}^{-/}$SCs comprised several neuronal or neuron differentiation genes, including birc5b, eloal, elavl2, htr2aa, and pou5f3, which were either unchanged or downregulated in uninjured $\mathrm{mstnh}^{-/-} \mathrm{SCs}$ (Fig. 6B-D). These findings indicated neuronal gene expression changes in mstnb mutants are injury-dependent.

Fibroblast growth factor (Fgf) maintains the proliferation and self-renewal capacities of neural stem cells in mammals (Hsu et al., 2009). By RNA-seq and qRT-PCR, fgf1b was downregulated in $m_{s t n b^{-/}}$SCs at 1wpi, but was unchanged in uninjured SC tissues (Fig. 6E). The dysregulation of fgf $1 b$ in mstnb mutants suggested mstnb-mediated fgf $1 b$ expression inhibits neurogenesis by promoting progenitor cell proliferation and self-renewal. To test this hypothesis, we examined whether the neuronal gene expression changes observed in mstnb mutants could be rescued by

262 localized delivery of human recombinant FGF1 into SC lesions. We injured $m s t n b^{-/-}$and wild-type 263 siblings and applied FGF1 proteins adjacent to the lesion site at 5 dpi using a gelfoam sponge. 264 Gene expression changes were assessed by qRT-PCR at $1 \mathrm{wpi}$, corresponding to 2 days after 265 treatment (Fig. 6F). Consistent with increased neurogenesis in mstnb mutants, birc5b, eloal, and 266 pou5f3 transcript levels were increased in vehicle-treated $m s t n b^{-/-}$relative to vehicle-treated wild267 types (Fig. 6G). Application of exogenous FGF1 proteins at the lesion rescued the upregulation 
of neuronal genes in mstnh $^{-/}$SCs (Fig. 6G). These findings indicated Mstn-mediated Fgf signaling

269 is a negative regulator of adult neurogenesis after zebrafish SCl.

\section{DISCUSSION}

270 This study shows mstnb expression is induced in a subset of dorsal ERGs after SCI. Our results

271 are consistent with a model in which mstnb regulates the rates of self-renewal and neuronal 272 differentiation after $\mathrm{SCl}$, and suggest $m s t n b$-dependent Fgf signaling promotes self-renewal at the 273 expense of neurogenesis (Fig. 6H).

Successful SC regeneration requires faithful recovery of the excitatory and inhibitory (E/I) balance in regenerating neural circuits. $\mathrm{SCl}$ alters the amount, strength, and relative locations of E/I inputs by disrupting descending hindbrain connections and promoting waves of axonal degeneration, neuronal death, and demyelination. Interneurons and motor neurons regenerate after zebrafish $\mathrm{SCl}$. Notably, dopamine and serotonin signals from regenerating tracts control motor neuron regeneration by promoting the proliferation of pMN ERGs (Barreiro-Iglesias et al., 2015; Reimer et al., 2013). We found mstnb mutants display increased neuronal differentiation at 1 wpi, with an overrepresentation in glycinergic interneurons among regenerating neurons. Glycinergic inhibition plays important roles in coordinating locomotor rhythms in different organisms (Hinckley et al., 2005; Jovanović et al., 1999; Sibilla and Ballerini, 2009). We propose that increased inhibitory neurotransmission disrupts $E / /$ balance and may underlie the behavioral recovery defects observed in mstnb mutants. The mechanisms that underlie E/I balance disruption in mstnb mutants require further investigation into the time course of neuronal regeneration and the contribution of $m s t n b^{+}$ERGs to specific neuronal populations.

Our study highlights a niche of dorsal ependymal progenitors that express mstnb after SCl. Unlike tissues that undergo constant cell renewal such as skin or blood, the nervous system undergoes little turnover and does not harbor a constitutively active neurogenic niche. Instead,

291 neural progenitors are quiescent and are only activated upon physiological or pathological 292 stimulation. Lineage restricted ependymal progenitors emerge after zebrafish SCI. ERG niches 293 include a ventro-lateral domain that gives rise to regenerating motor neurons, and a ventral 294 domain that undergoes epithelial-to-mesenchymal transition and is required for glial bridging after 295 SCl (Klatt Shaw et al., 2021; Reimer et al., 2008). Our findings support the emergence of a lineage 296 restricted, neurogenic niche of dorsal ERGs during SC regeneration in zebrafish. Consistent with 297 this model, the numbers of motor neurons and the extent of glial bridging across the lesion, which 
have been respectively associated with ventro-lateral and ventral ERGs, are unaffected in the absence of mstnb. Instead, mstnb mutants showed specific neurogenesis defects in dorsal SCs, and a preferential increase in dorsal glycinergic neurons. The molecular identity and cellular contributions of $m s t n b^{+}$ERGs to neurogenesis and SC repair warrant further investigation. renewal, differentiation, and quiescence ( $\mathrm{Li}$ and Clevers, 2010). At the cellular level, the organization of progenitor cells into localized niches maintains quiescence under homeostatic conditions, and triggers progenitor cell activation following niche disruption (Bagheri-Mohammadi, 2021). Molecularly, progenitor cell niches are hubs for Bmp, Wnt, and Notch signaling pathways, which control the rates of self-renewal, differentiation, and quiescence. Our study supports a model in which Mstn restricts neuronal differentiation and maintains neuronal progenitors in a proliferative, undifferentiated cell fate. Our findings are consistent with previously reported functions for Mstn in muscle, fat, and bone tissues (Dogra et al., 2017; Langley et al., 2002; Le and Yao, 2017; Lim et al., 2018; McCroskery et al., 2003; Wallner et al., 2017). Notably, previous findings have shown that Mstn is a regeneration limiting gene for zebrafish heart or fin regeneration, which are both dedifferentiation-based repair mechanisms (Dogra et al., 2017;

314 Magga et al., 2019; Uribe et al., 2018). In contrast, in the context of SC regeneration, we find that 315 Myostatin promotes regeneration by supporting regenerative FGF signaling, revealing a new role 316 for Myostatin in this stem cell-based regeneration paradigm. Together these findings underline 317 how tissue regeneration programs can coopt similar signaling pathways to achieve highly specific 318 regenerative outcomes, and indicate a tissue-specific mechanism for Myostatin signaling.

We propose that Fgf is a mediator of Mstn functions during SC regeneration, and that Mstn limits neuronal differentiation by promoting Fgf-dependent self-renewal in $m s t n b^{+}$ERGs. Similar regulatory mechanisms have been shown in muscle tissues, where Mstn inhibits the muscle differentiation transcription factors MyoD and Myogenin. Fgf signaling promotes NSC proliferation and self-renewal (Hsu et al., 2009). In mammals, isolated ependymal cells have the capacity to

324 form neurospheres and produce neurons, astrocytes and oligodendrocytes in vitro (Meletis et al., 325 2008). However, although mammalian SCl induces the proliferation of ependymal cells lining the 326 central canal (Horner et al., 2000), mammalian ependymal cells are incapable of forming neurons 327 in vivo (Barnabe-Heider et al., 2010; Muthusamy et al., 2018; Ren et al., 2017; Shah et al., 2018). 328 We propose that comparative studies between zebrafish ERGs and mammalian ependymal cells 
bioRxiv preprint doi: https://doi.org/10.1101/2021.08.18.456778; this version posted August 19, 2021. The copyright holder for this preprint (which was not certified by peer review) is the author/funder. All rights reserved. No reuse allowed without permission.

329 could reveal new insights into their differential regenerative capacities and examine whether Mstn 330 signaling is differentially regulated between zebrafish and mammals. 


\section{ACKNOWLEDGMENTS}

331 We thank V. Cavalli, A. Johnson, K. Poss, and L. Solnica-Krezel for discussion, D. Stainier for 332 sharing mstnb mutants, T. Li and B. Zhang for Bioinformatics analysis, and the Washington 333 University Zebrafish Shared Resource for animal care. This research was supported by grants 334 from the NIH (R01 NS113915 to M.H.M.) and the McDonnell Center for Cellular Neuroscience (to 335 M.H.M.). 
Figure 1
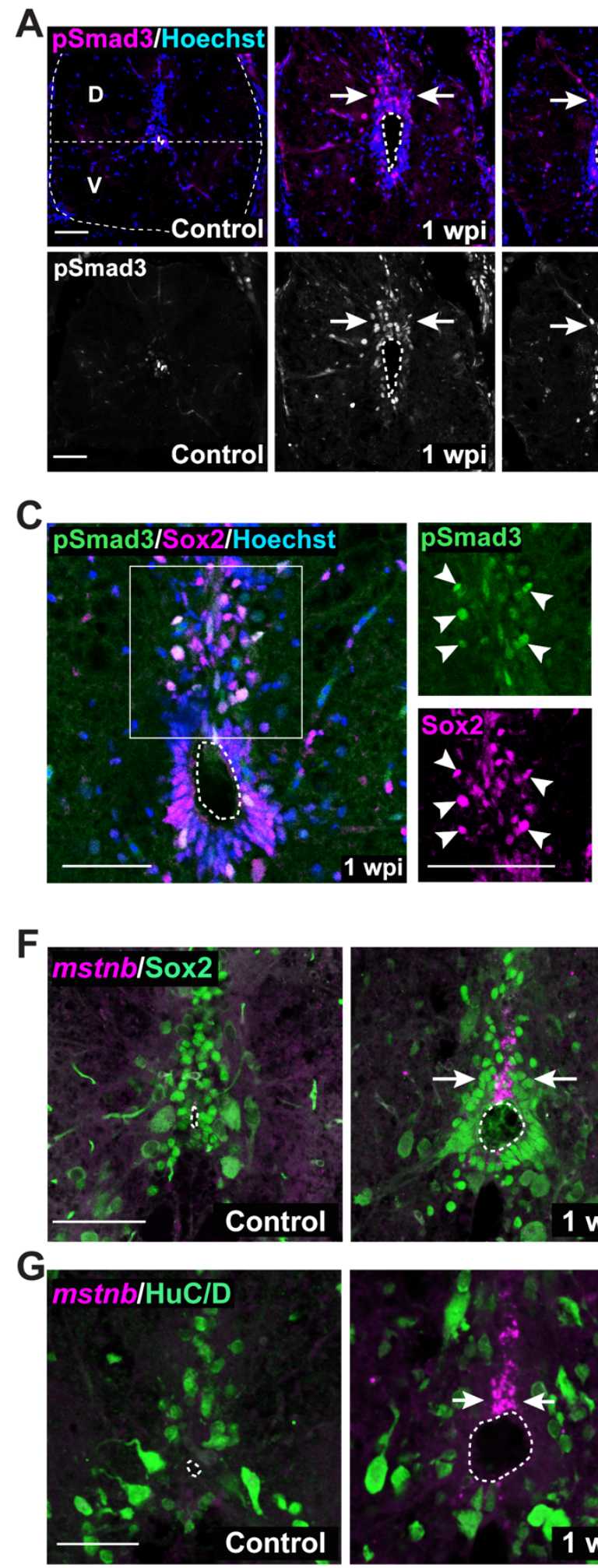

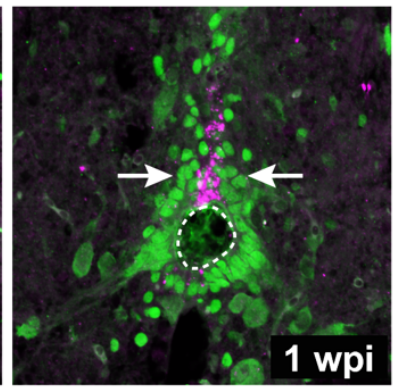

D
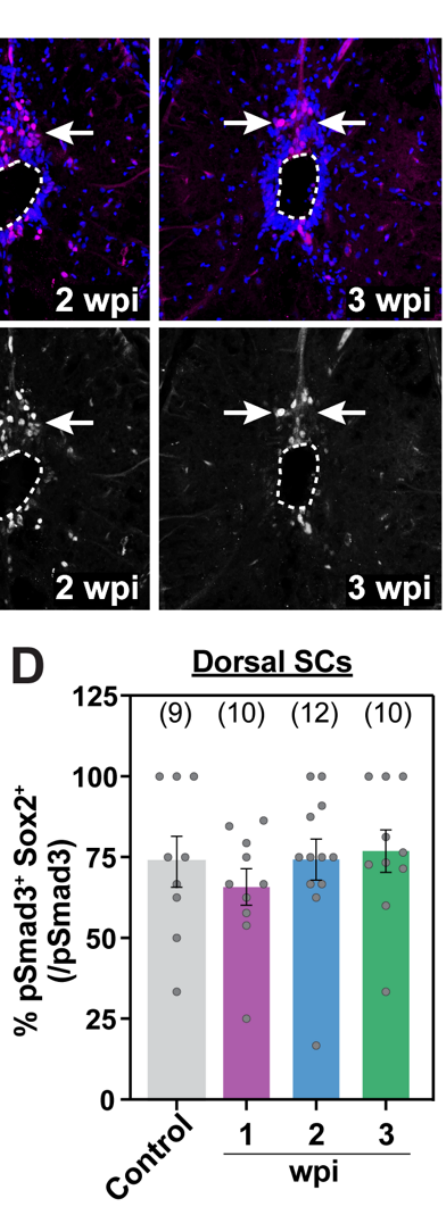

B
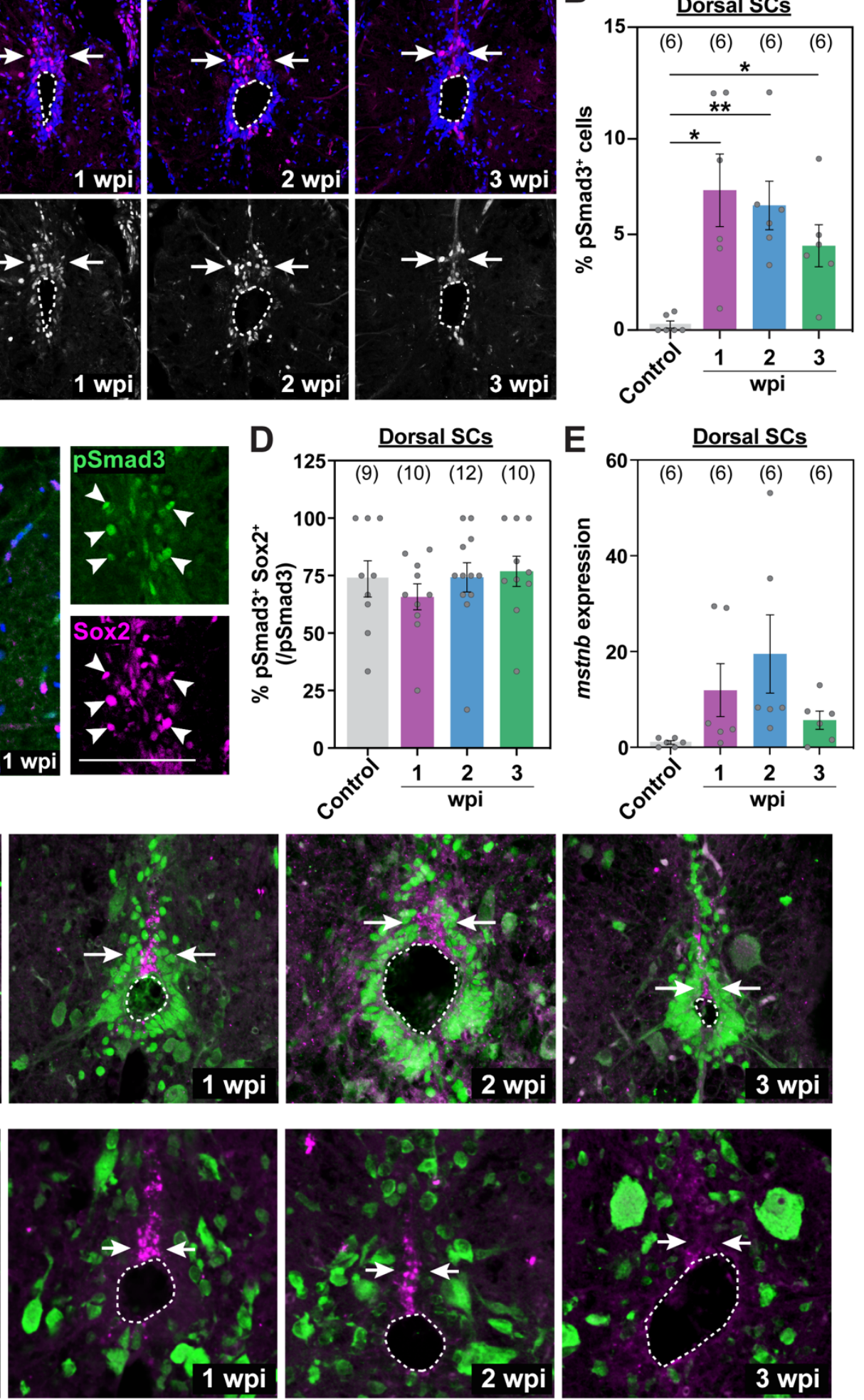
336 Figure 1. mstnb is induced in dorsal ependymal progenitors during SC regeneration. (A) 337 Immunostaining for phosphorylated Smad3 (pSmad3) after SCI. Wild-type SC sections at 1, 2, 338 and $3 \mathrm{wpi}$, and uninjured controls are shown. Cross sections $450 \mu \mathrm{m}$ from the lesion site are 339 shown. Horizontal dotted line demarcates dorsal (D) and ventral (V) SC domains. Arrows point to $340 \mathrm{pSmad}^{+}$nuclei in the dorsal domain. (B) pSmad3 quantification in dorsal sections of wild-type 341 SCs. Percent pSmad3 ${ }^{+}$cells was normalized to the number of nuclei in dorsal SCs. (C) pSmad3 342 and Sox2 immunostaining in wild-type SCs at 1 wpi. High-magnification views of dorsal SCs are 343 shown. Arrowheads point to pSmad3 ${ }^{+}$Sox2 ${ }^{+}$ERGs. (D) pSmad3 quantification in dorsal ERGs.

344 Percent $p S m a d 3^{+}$Sox $2^{+}$cells was normalized to the number of $p S m a d 3^{+}$cells. (E) Quantification 345 of mstnb by in situ hybridization in dorsal SC tissues. (F,G) mstnb expression in wild-type SC 346 sections after SCl. mstnb fluorescence in situ hybridization was followed by immunostaining for 347 either Sox2 (F) or HuC/D (G) antibodies. Cross sections $450 \mu \mathrm{m}$ from the lesion site are shown at 3481,2 , and $3 \mathrm{wpi}$, and for uninjured controls. Arrows point to domains of $m s t n b$ expression in dorsal 349 SCs. Dotted ovals delineate central canal edges. For all quantification, SC sections $450 \mu \mathrm{m}$ rostral 350 to the lesion were analyzed and sample sizes are indicated in parentheses. ${ }^{*} P<0.05 ;{ }^{*} P<0.01$. 351 Scale bars, $50 \mu \mathrm{m}$. 
Figure 2

A

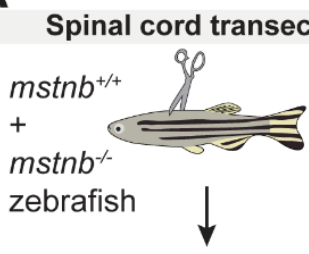

Functional recovery swim endurance

+ swim behavior assays
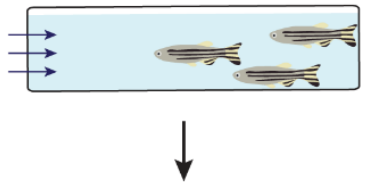

Glial bridging + Axon tracing (histology)

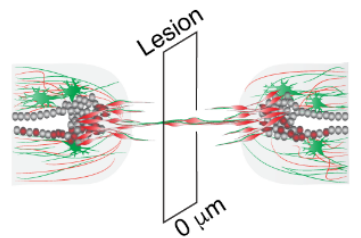

E

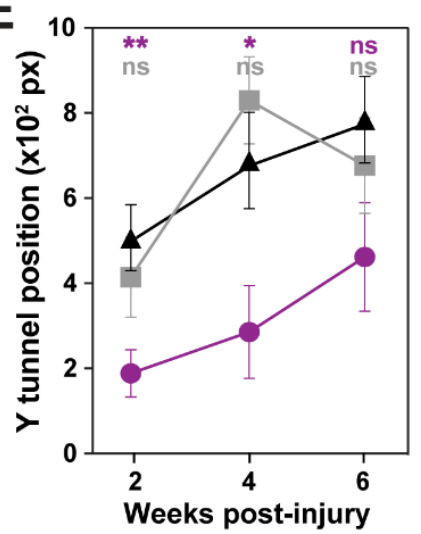

B

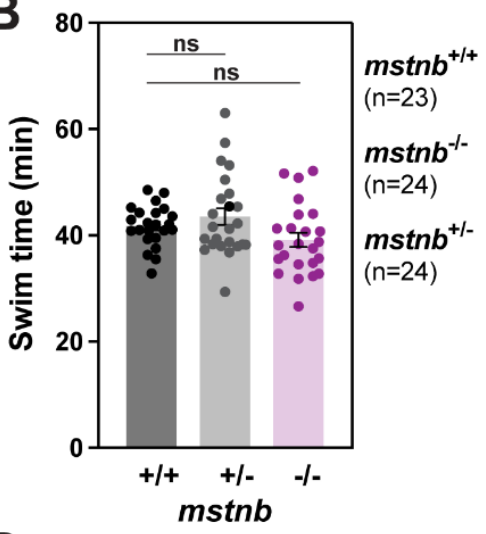

$\mathbf{D}$

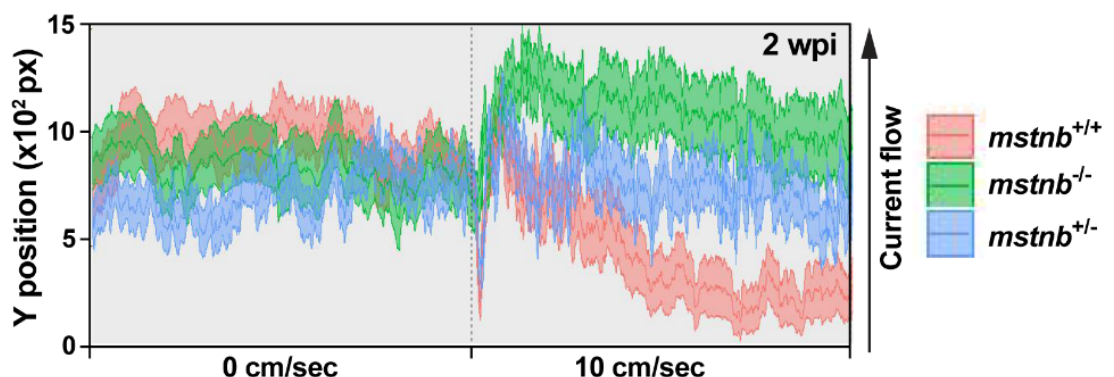

C

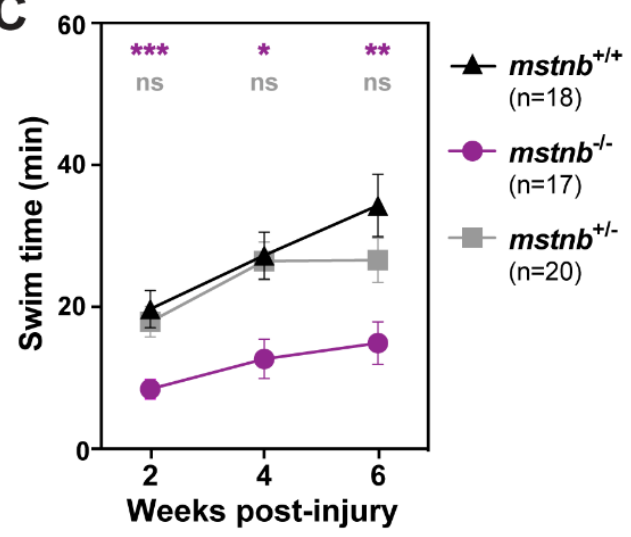

H
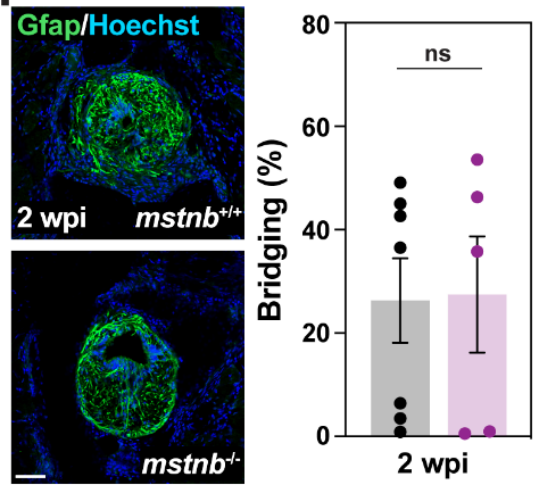

I

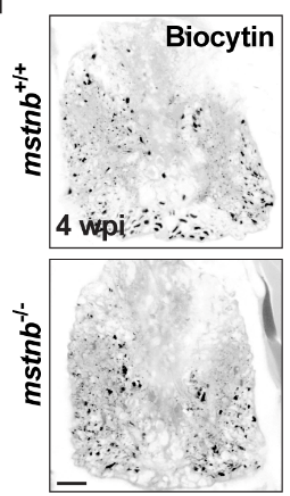

G
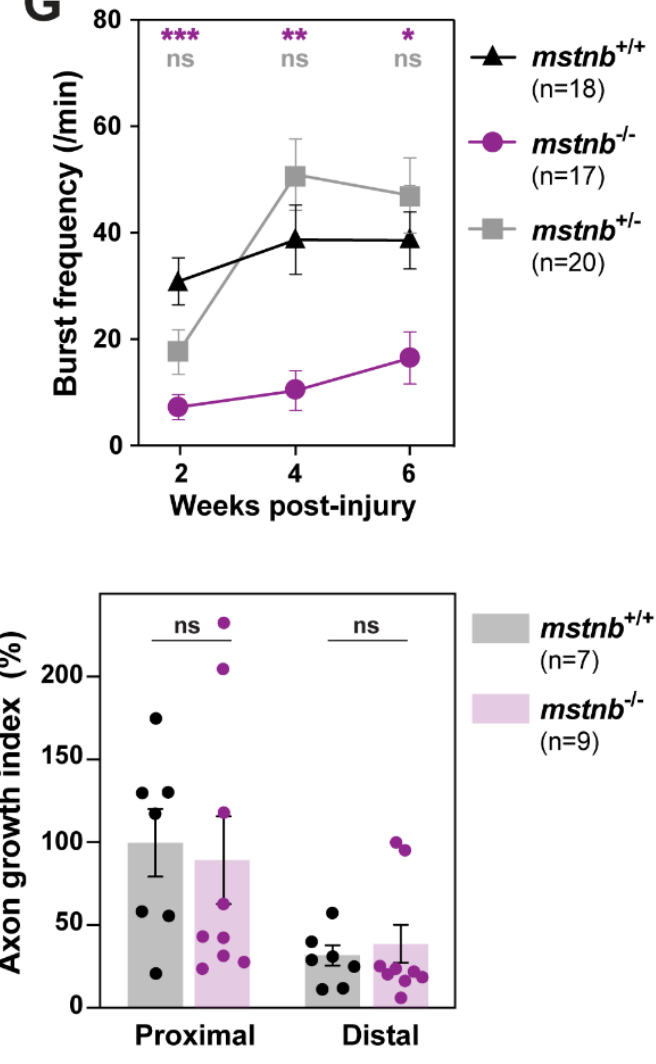
353 Figure 2. mstnb is required for functional SC regeneration. (A) Experimental pipeline to 354 examine regeneration phenotypes after SCI. mstnh $^{-/}$fish and wild-type siblings were subjected 355 to complete SC transection. Functional recovery was assessed between 2 and 6 wpi. Histology 356 was used to assess glial and axonal bridging at 2 and 4 wpi, respectively. (B) Endurance swim 357 assays determined baseline motor function for $m s t n b^{-/+}, m s t n b^{-/}$, and wild-type fish. Dots 358 represent individual animals from three independent clutches. (C) Endurance swim assays for $359 \mathrm{mstnh}^{-/+}, \mathrm{mstnh}^{-/}$, and wild-type fish at 2, 4, and 6 wpi. Dots represent individual animals from two 360 independent experiments. Statistical analyses of swim times are shown for $m s t n b^{-/+}$(grey) and $361 \mathrm{mstnh}^{-/}$(magenta) relative to wild types. Recovery of $m s t n b^{-/}$animals was not significant between 3622 and 6 wpi. (D) Tracking swim performance at minimal water current velocity for $m s t n b^{-/+}$(blue), $363 \mathrm{mstnh}^{-/}$(green), and wild-type siblings (red). Average $\mathrm{Y}$ position is shown for each cohort at 2 wpi.

364 Animals were tracked in the absence of current $(0 \mathrm{~cm} / \mathrm{sec})$ for $5 \mathrm{~min}$, and for a $10 \mathrm{~cm} / \mathrm{sec}$ current 365 velocity for $5 \mathrm{~min}$. The arrow shows the direction of the water current. (E-G) Average $Y$ position 366 in the tunnel $(E)$, percent activity $(F)$, and burst frequency $(G)$ were quantified at $10 \mathrm{~cm} / \mathrm{sec}$ water 367 current velocity. $m s t n b^{-/+}, m s t n b^{-/}$, and wild-type fish are shown at 2, 4, and 6 wpi. Statistical 368 analyses of swim times are shown for $m s t n b^{-/+}$(grey) and $m s t n b^{-/-}$(magenta) relative to wild types.

369 Two independent experiments are shown. (H) Glial bridging in stn $^{-/-}$(magenta) and wild-type

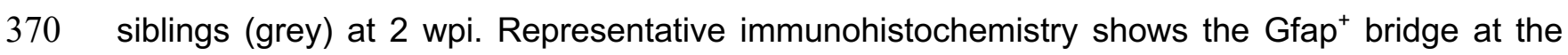
371 lesion site. Percent bridging represents the cross-sectional area of the glial bridge at the lesion 372 site relative to the the intact SC. Percent bridging was quantified for 7-9 animals per group. (I) 373 Anterograde axon tracing in in $\mathrm{mstnb}^{-/}$(magenta) and wild-type zebrafish (grey) at 4 wpi. Biocytin 374 axon tracer was applied rostrally and analyzed at $100 \mu \mathrm{m}$ (proximal) and $500 \mu \mathrm{m}$ (distal) caudal 375 to the lesion. Representative traces of biocytin are shown for each genotype animals at the 376 proximal level. Quantification represents 7-9 animals per group. Axon growth was normalized to 377 Biocytin labeling in wild-types at the proximal level. ${ }^{*} \mathrm{P}<0.05$; ${ }^{* *} \mathrm{P}<0.01$; ${ }^{* *} \mathrm{p}<0.001$; ns, not 378 significant. Scale bars, $50 \mu \mathrm{m}$. 
Figure 3

A

Cell proliferation in mstnb mutants

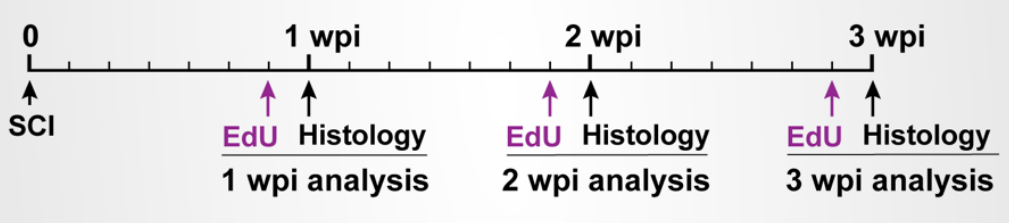

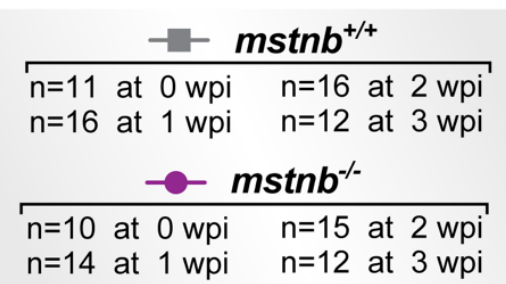

C

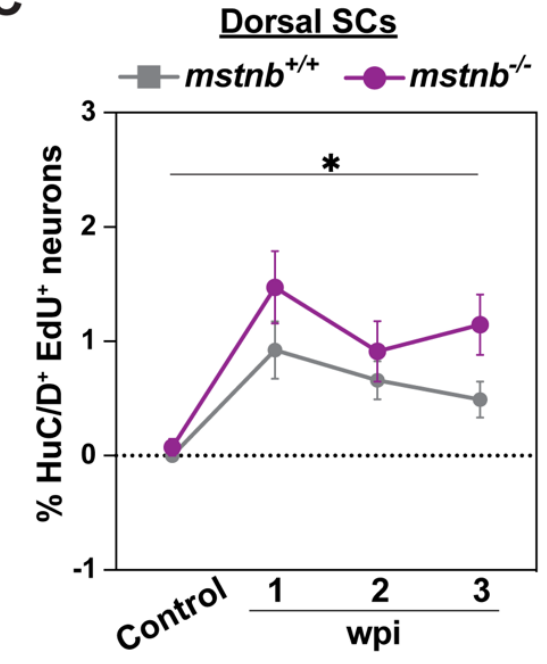

$E$

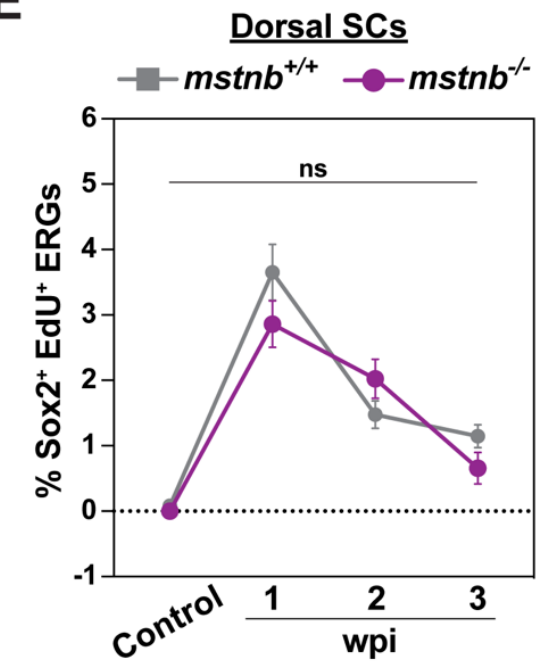


379 Figure 3. Cell proliferation in mstnb mutant zebrafish. (A) Experimental timeline to assess the 380 rates of cell proliferation. $m s t n b^{-/}$and wild-type siblings were subjected to SC transections. A 381 single EdU injection was performed at either 6, 13, or 20 days post-injury. SC tissues were 382 harvested for analysis at 1, 2, 3 wpi and at 24 hours after EdU injection. Animal numbers are 383 indicated for each genotypes and two independent replicates are shown. (B) 384 Immunohistochemistry for EdU and HuC/D in SC sections of $m s t n b^{+/+}$and $m s t n b^{-/}$at 1 wpi. The 385 region inside the rectangular box is shown in higher magnification. Arrowheads indicate $\mathrm{HuC} / \mathrm{D}^{+}$

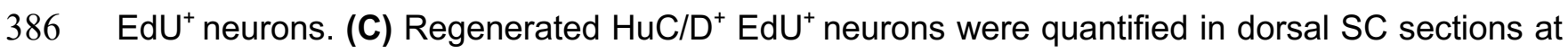
387 1, 2, 3 wpi and uninjured controls. Percent HuC/D+ EdU ${ }^{+}$neurons was normalized to the total 388 number of nuclei for each section. (D) Immunohistochemistry for EdU and Sox2 in SC sections of $389 \mathrm{mstnb}^{+/+}$and $m s t n b^{-/}$at 1 wpi. The region inside the rectangular box is shown in higher 390 magnification. Arrowheads indicate Sox2 ${ }^{+} \mathrm{EdU}^{+} \mathrm{ERGs}$. (E) Sox2 ${ }^{+} \mathrm{EdU}^{+} \mathrm{ERGs}$ were quantified in 391 dorsal SC sections at 1, 2, 3 wpi and uninjured controls. Percent Sox2 ${ }^{+} \mathrm{EdU}^{+}$ERGs was 392 normalized to the total number of nuclei for each section. For all quantifications, cross SC sections $393450 \mu \mathrm{m}$ rostral to the lesion site were quantified. ${ }^{*} \mathrm{P}<0.05$; ns, not significant. Scale bars, $50 \mu \mathrm{m}$. 394 Dotted ovals delineate central canal edges. 
Figure 4

A
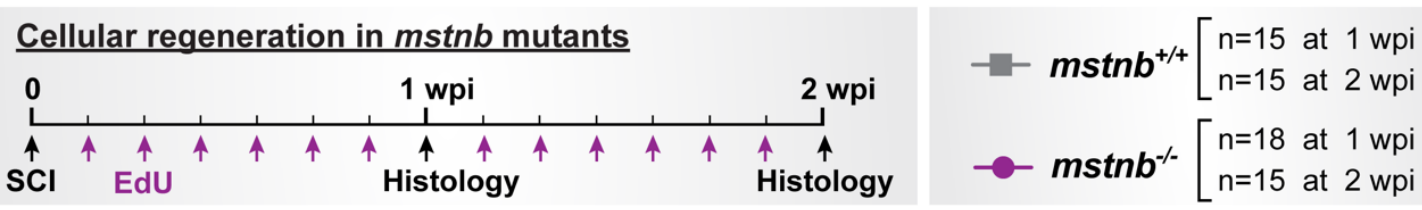

B

E

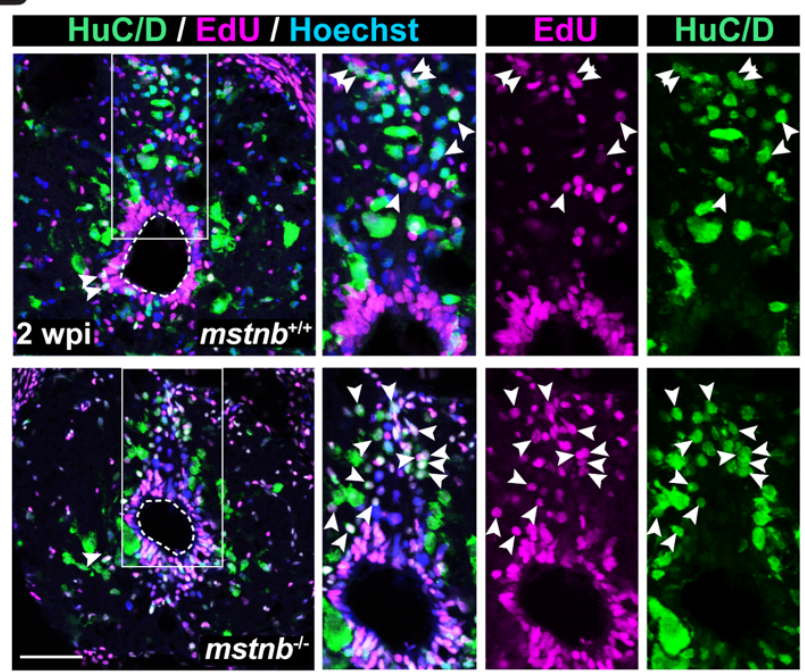

C

Dorsal SCs

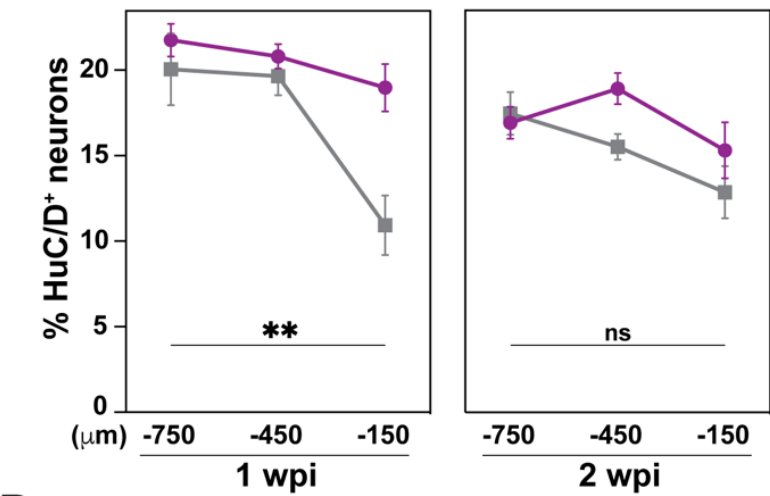

D
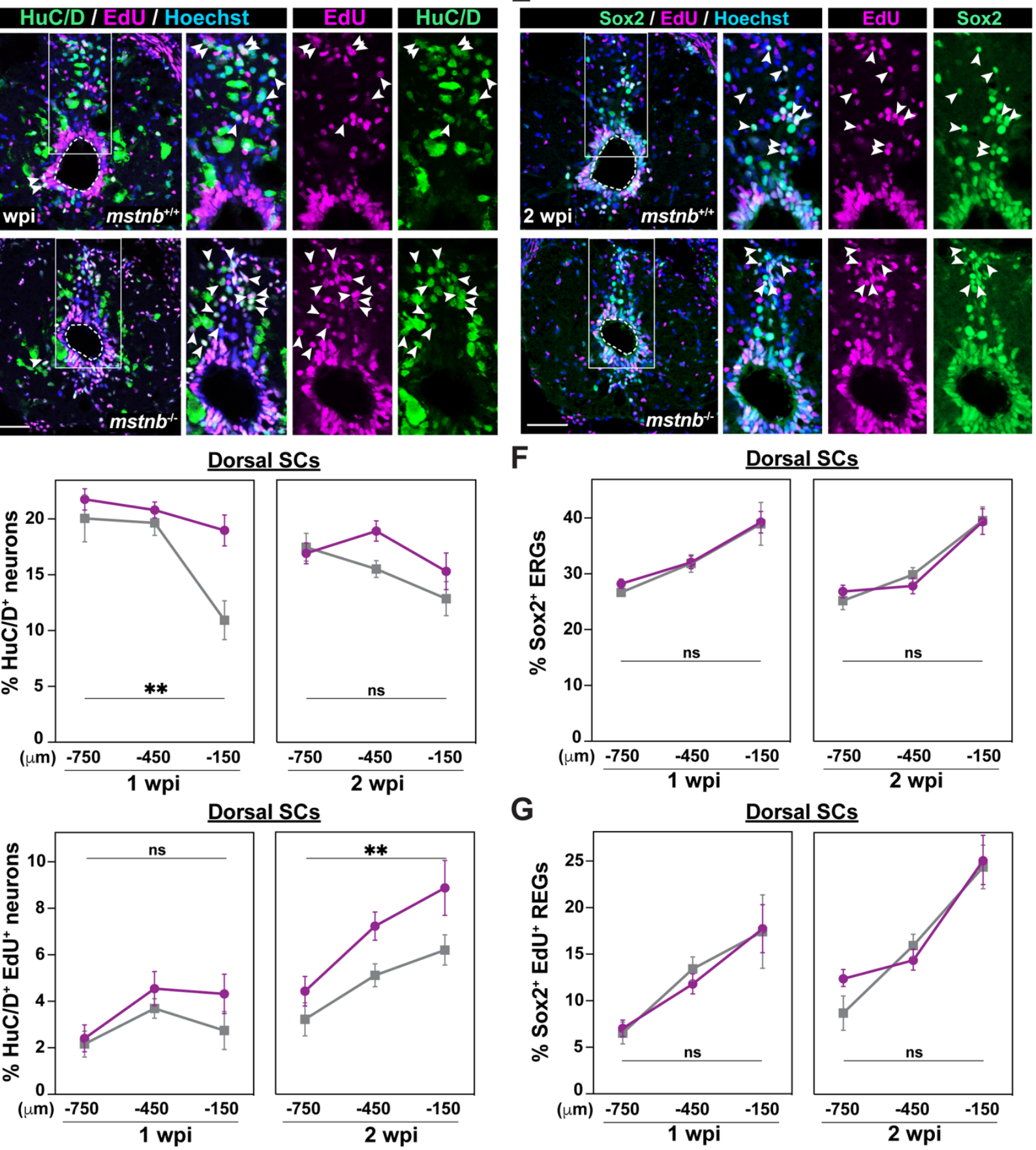

$\mathbf{F}$

Dorsal SCs

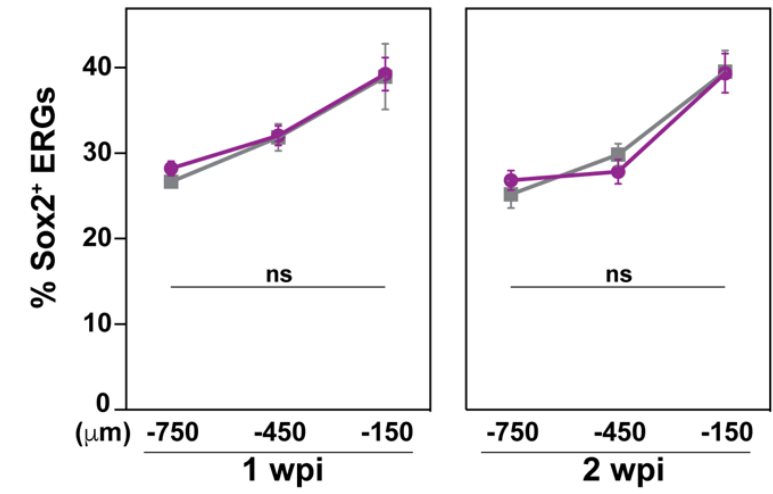

G

Dorsal SCs
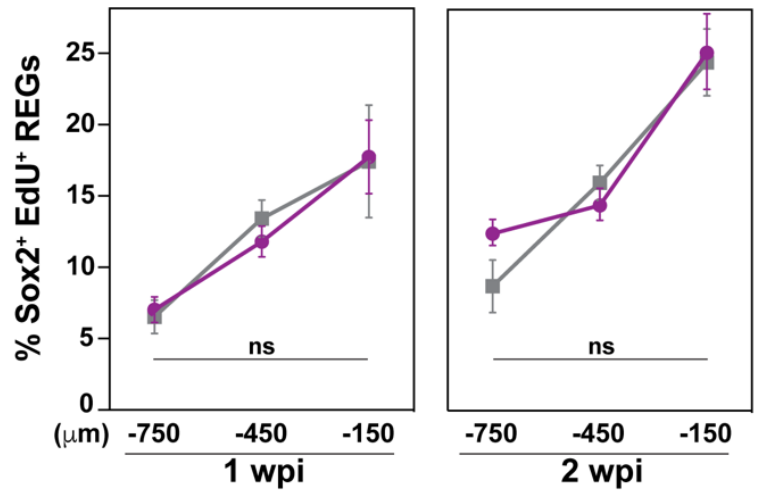
395 Figure 4. Regenerative neurogenesis in mstnb mutant zebrafish. (A) Experimental timeline

396 to assess the rates of neurogenesis and ERG self-renewal. $m s t n b^{-/}$and wild-type siblings were 397 subjected to SC transections and daily EdU injections. SC tissues were harvested for analysis at 3981 or 2 wpi. Animal numbers are indicated for each genotypes and two independent replicates are 399 shown. (B) Immunohistochemistry for EdU and HuC/D in SC sections of $m s t n b^{+/+}$and $m s t n b^{-/}$at 4002 wpi. The region inside the rectangular box is shown in higher magnification. Arrowheads indicate $401 \mathrm{HuC} / \mathrm{D}^{+} E \mathrm{dU}{ }^{+}$neurons. (C) HuC/D+ neurons were quantified in dorsal SC sections at 1 and 2 wpi. 402 Percent $\mathrm{HuC} / \mathrm{D}^{+}$neurons was normalized to the total number of nuclei for each section. (D) 403 Regenerated $\mathrm{HuC} / \mathrm{D}^{+} \mathrm{EdU}^{+}$neurons were quantified in dorsal SC sections at 1 and 2 wpi. Percent $404 \mathrm{HuC} / \mathrm{D}^{+} \mathrm{EdU}^{+}$neurons was normalized to the total number of nuclei for each section. (E) 405 Immunohistochemistry for EdU and Sox2 in SC sections of $m s t n b^{+/+}$and $m s t n b^{-/}$at 2 wpi. The 406 region inside the rectangular box is shown in higher magnification. Arrowheads indicate Sox $2^{+}$ 407 EdU ${ }^{+}$ERGs. (F) Sox2 ${ }^{+}$ERGs were quantified in dorsal SC sections at 1 and 2 wpi. Percent Sox2 ${ }^{+}$ 408 ERGs was normalized to the total number of nuclei for each section. (G) Sox2 ${ }^{+}$EdU $^{+}$ERGs were 409 quantified in dorsal SC sections at 1 and 2 w. Percent Sox2 ${ }^{+}$EdU ERGs was normalized to the 410 total number of nuclei for each section. For all quantifications, cross SC sections at 150, 450, and $411750 \mu \mathrm{m}$ rostral to the lesion site were quantified. ${ }^{* *} \mathrm{P}<0.01$; ns, not significant. Scale bars, $50 \mu \mathrm{m}$. 412 Dotted lines delineate central canal edges. 
A
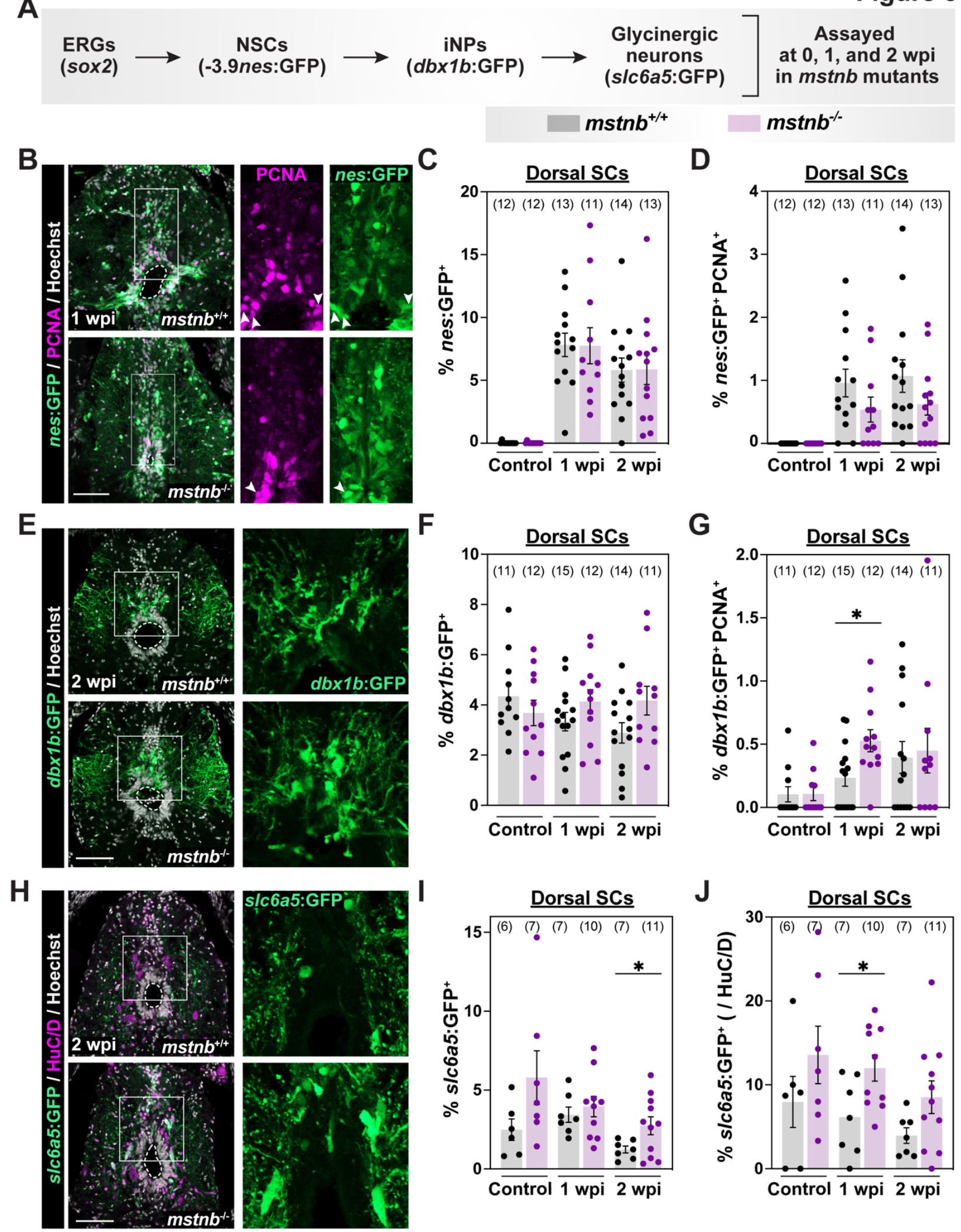
413 Figure 5. Assessment of neuronal progenitors and neurons in mstnb mutant zebrafish. (A)

414 Experimental timeline to elucidate the dynamics of neurogenesis. $\mathrm{mstnh}^{-/}$animals were crossed 415 into either-3.9nestin:GFP, $d b \times 1 b$ :GFP, or s/c6a5:GFP to evaluate the numbers of neural stem 416 cells (NSCs), intermediate neural progenitors (iNPs), and glycinergic neurons, respectively. $417 \mathrm{mstnh}^{-/}$and wild-type siblings were subjected to SC transections and collected at 1 or 2 wpi for 418 analysis. Uninjured controls were used. Animal numbers are indicated for each genotypes and 419 two independent replicates are shown. (B) GFP and PCNA staining in -3.9nestin:GFP; $m s t n b^{-/}$SC 420 sections at 1 wpi. -3.9nestin:GFP; $m s t n b^{+/+}$siblings are used as controls. The region inside the 421 rectangular box is shown in higher magnification. (C) nes ${ }^{+}$NSCs were quantified in dorsal SC 422 sections. Percent $n e s^{+}$NSCs was normalized to the total number of nuclei for each section. (D) $423 n^{+} \mathrm{PCNA}^{+}$NSCs were quantified in dorsal SC sections. Percent nes ${ }^{+} \mathrm{PCNA}^{+} \mathrm{NSCs}$ was 424 normalized to the total number of nuclei for each section. (E) GFP staining in $d b x 1 b$ :GFP; $m s t n b^{-}$ 425 /- SC sections at 2 wpi. $d b \times 1 b: G F P ; m s t n b^{+/+}$siblings are used as controls. The region inside the rectangular box is shown in higher magnification. (F) $d b \times 1 b^{+}$iNPs were quantified in dorsal SC sections. Percent $d b \times 1 b^{+}$iNPs was normalized to the total number of nuclei for each section. (G) $d b \times 1 b^{+} \mathrm{PCNA}^{+}$NSCs were quantified in dorsal SC sections. Percent $d b \times 1 b^{+} \mathrm{PCNA}^{+}$iNPs was normalized to the total number of nuclei for each section. (H) GFP staining in slc6a5:GFP:GFP; $m s t n b^{-/}$SC sections at 2 wpi. slc6a5:GFP:GFP; $m s t n b^{+/+}$siblings are used as

431 controls. The region inside the rectangular box is shown in higher magnification. (I) s/c6a5:GFP 432 glycinergic neurons were quantified in dorsal SC sections. Percent slc6a5:GFP ${ }^{+}$neurons was 433 normalized to the total number of nuclei for each section. (J) Percent slc6a5:GFP ${ }^{+}$neurons was

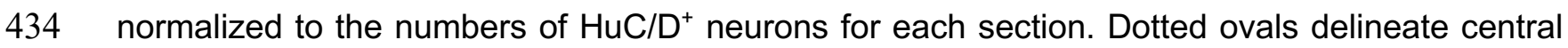
435 canal edges. For all quantifications, cross SC sections $450 \mu \mathrm{m}$ rostral to the lesion site were 436 quantified. ${ }^{*} \mathrm{P}<0.05$; ns, not significant. Scale bars, $50 \mu \mathrm{m}$. 
Figure 6

A

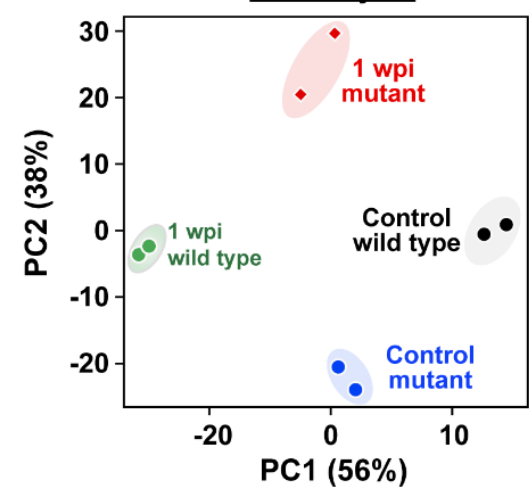

D

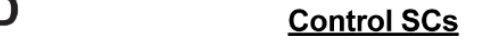

B

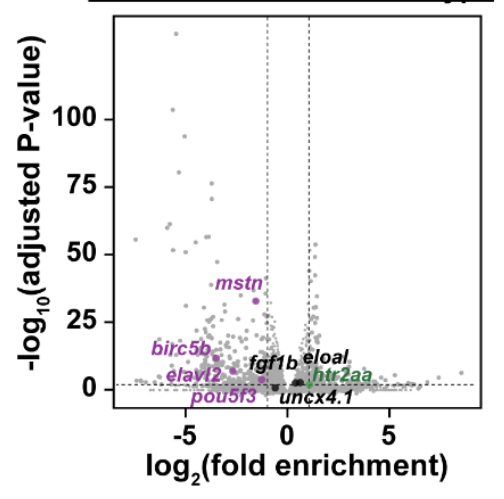

C

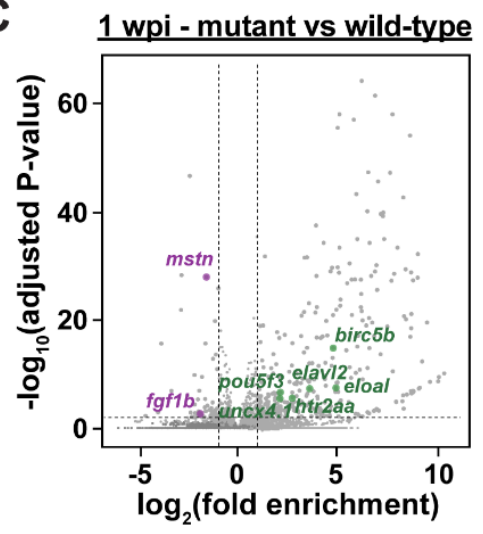

E
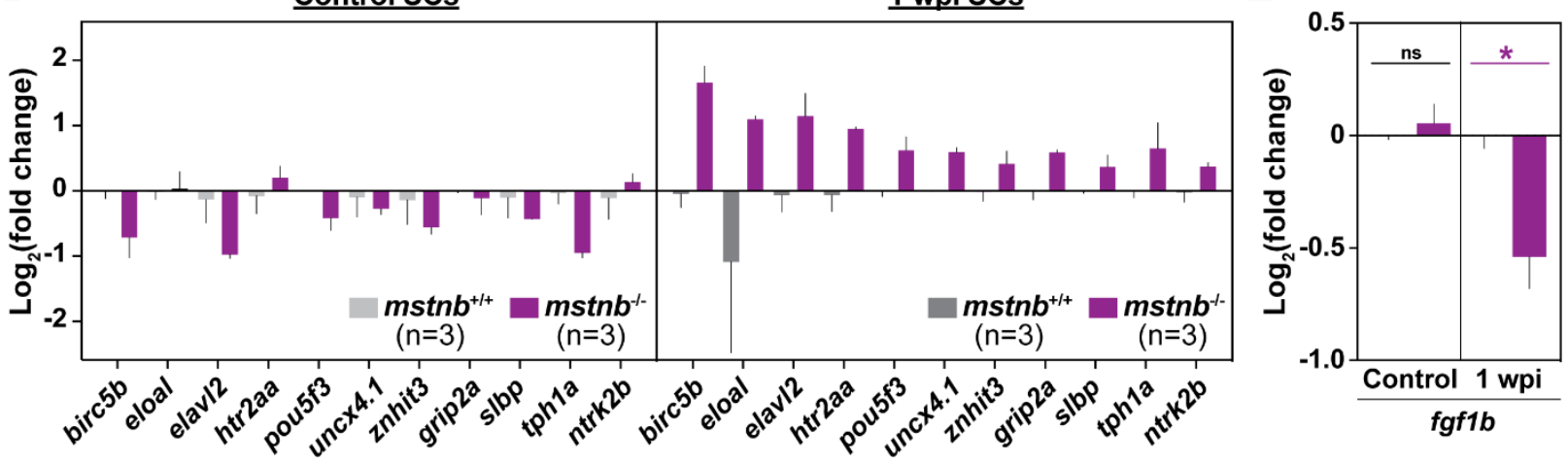

F

FGF1 treatment in mstnb mutants
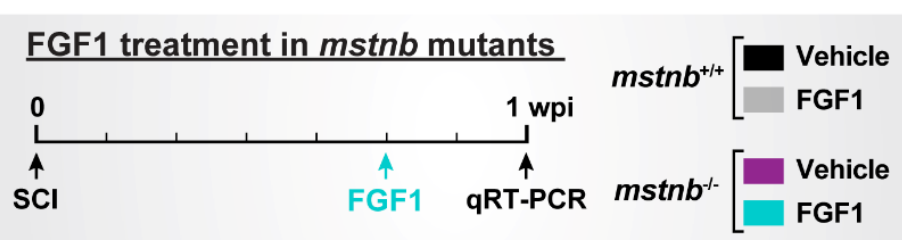

H

G 1 wpi SCs
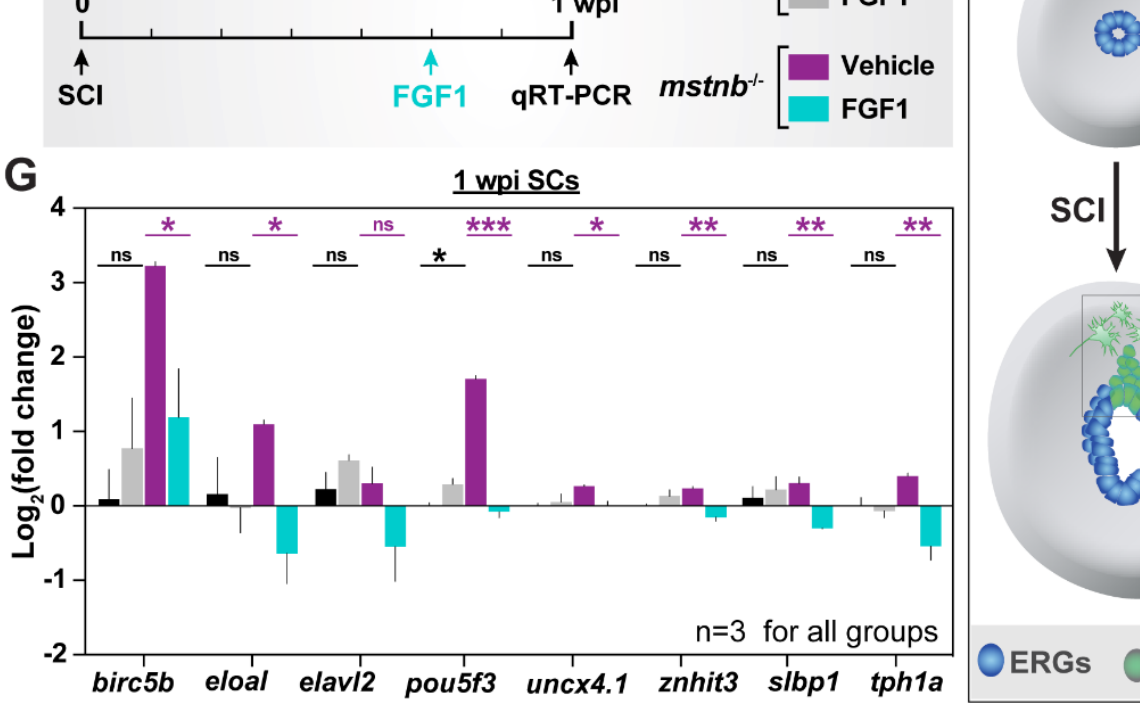

83

Dorsal

Ventral

Interneurons (huc, glyt2)

$\uparrow$

iNPs

(dbx1b)

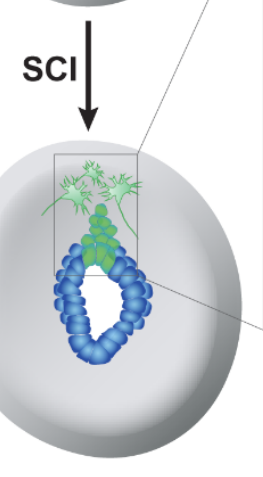

$\underset{\substack{\text { NSCstin) } \\ \uparrow}}{\substack{\text { dERGs } \\(\text { sox2) }}}$

fgf1b

mstnb

OERGs Omstnb+ERGs Interneurons 
Figure 6. mstnb regulates neuronal gene expression via fgf1b. (A) $m s t n b^{-/}$and wild-type

438 siblings were subjected to complete SC transections and collected at 1 wpi for RNA sequencing.

439 Control SC tissues were collected from uninjured fish. Sequencing was performed in independent

440 duplicates. Principle component analysis shows clustering of biological replicates. Principle

441 components 1 and 2 (PC1 and PC 2) show 56\% and 38\% variance, respectively. (B,C) Volcano

442 plot representation of genes that are significantly upregulated or downregulated or depleted in

$443 \mathrm{mstnh}^{-/}$SCs relative to wild-type controls. Upregulated genes included genes with $\log _{2}$ (fold

444 enrichment) $>1$ and adjusted $P$-value $<0.01$. Downregulated genes included genes with $\log _{2}$ (fold

445 enrichment) $<-1$ and adjusted $\mathrm{P}$-value $<0.01$ are considered downregulated. Select upregulated

446 (green) and downregulated (magenta) neuronal genes are indicated. Unchanged genes are

447 labelled in black. (D) qRT-PCR for neuronal genes was performed on mstnh $^{-/}$and wild-type SCs

448 at 1 wpi. Uninjured $m s t n b^{-/}$and wild-type controls were used. For each time point, $\log _{2}$ (fold

449 change) was normalized to eif1 $\alpha$ and to gene expression levels in sstn $^{+/+}$controls. (E) fgf1b

450 qRT-PCR was performed on uninjured and injured $m s t n b^{-/}$and wild-type animals. For each time

451 point, fgf1 $b$ expression was normalized to eif1 $\alpha$ as a loading control and to $f g f 1 b$ levels in $m s t n b^{+/+}$

452 controls. (F) mstn $^{-/-}$and wild-type siblings were subjected to SC transections and treated with

453 gelfoam-soaked human recombinant FGF1 at $5 \mathrm{dpi}$. $\mathrm{mstnh}^{-/}$and wild-type controls were treated

454 with vehicle-soaked gelfoam. SCs were collected for gene expression analysis at 1 wpi, which

455 corresponds to 2 days post-treatment. Animal numbers are indicated for each genotype. (G)

456 Neuronal gene expression was analyzed by qRT-PCR from FGF1- and vehicle-treated SC

457 tissues. For each gene, $\log _{2}$ (fold change) was normalized to eif1 $\alpha$ as a loading control and to 458 gene expression levels in uninjured $m s t n b^{+/+}$SCs. (H) Schematic model shows $m s t n b$ is a 459 negative regulator of neuronal differentiation in dorsal SC tissues after spinal cord injury. ERGs 460 (blue) undergo extensive proliferation after SCl. A mstnb expressing niche (green) emerges in 461 the dorsal ependyma. 


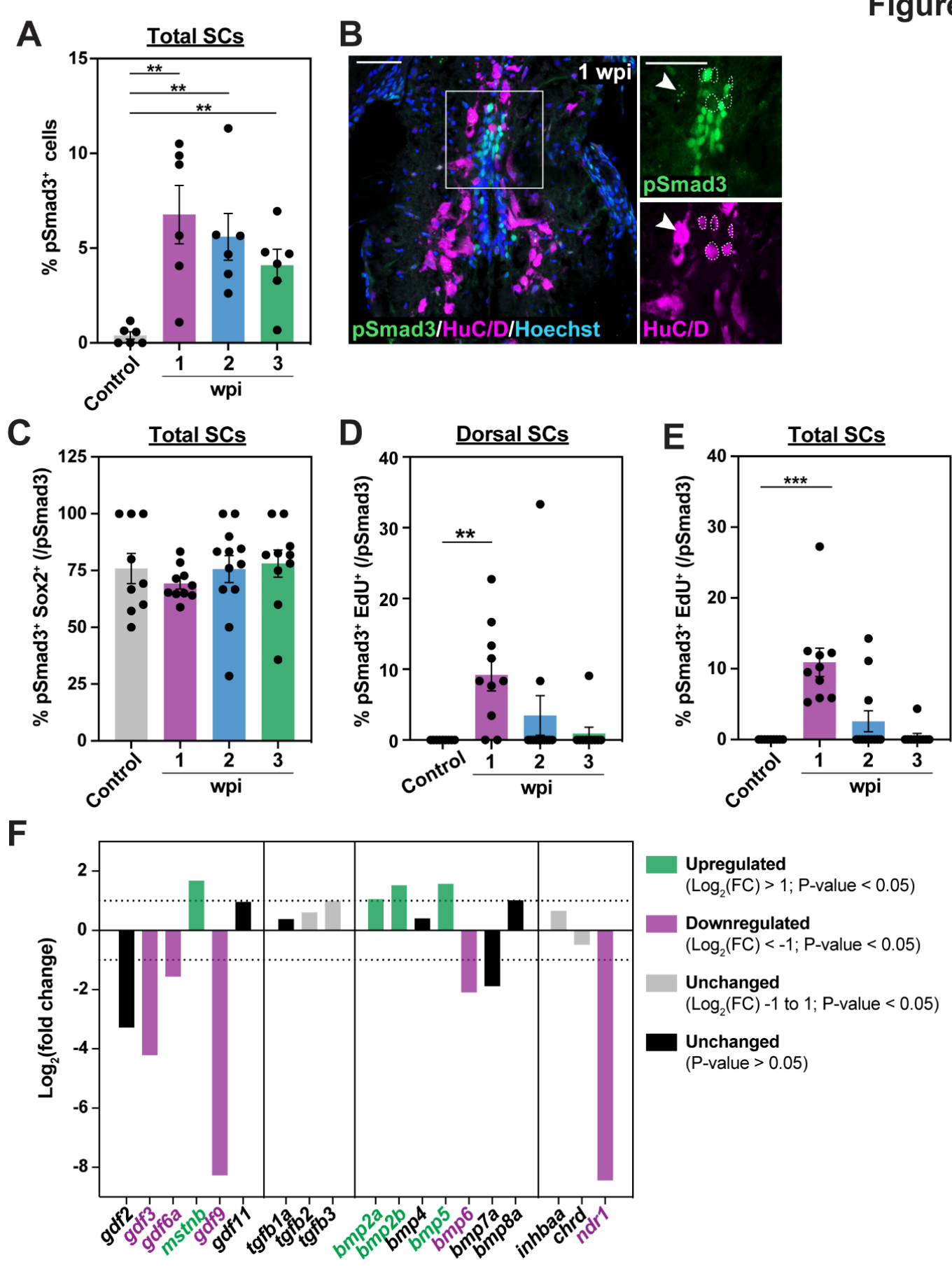


462 Figure S1. TGF- $\beta$ signaling during SC regeneration. (A) pSmad3 quantification in total SC

463 sections. Wild-type SCs at 1, 2 and 3 wpi and uninjured SCS were analyzed. Percent pSmad $3^{+}$

464 cells was normalized to the number of nuclei in dorsal SCs. (B) pSmad3 and HuC/D

465 immunostaining in wild-type SCs at 1 wpi. High-magnification views of dorsal SCs are shown.

466 Dotted lines delineate $\mathrm{pSmad} 3^{-} \mathrm{HuC} \mathrm{D}^{+}$neurons. Arrowheads point to dotted pSmad3 expression

467 in a subset of $\mathrm{HuC}^{+} \mathrm{D}^{+}$neurons. (C) pSmad3 quantification in total ERGs. Wild-type SCs at 1, 2

468 and 3 wpi and uninjured SCS were analyzed. Percent pSmad3 ${ }^{+}$Sox ${ }^{+}$cells was normalized to

469 the number of pSmad3 ${ }^{+}$cells. (D, E) pSmad3 and EdU quantification in dorsal (D) and total (E)

470 SCs. EdU was administered for $24 \mathrm{hrs}$ prior to SC collection. Wild-type SCs at 1, 2 and 3 wpi and

471 uninjured SCS were analyzed. Percent $\mathrm{pSmad}^{+} \mathrm{EdU}^{+}$cells was normalized to the number of

472 pSmad3 ${ }^{+}$cells. (F) Expression of Tgf- $\beta$ ligands in wild-type SCs at 2 wpi by bulk RNA sequencing.

473 For each gene, $\log _{2}$ (fold change) was normalized to gene expression levels in sham injured SCs.

474 Upregulated (green) and downregulated (magenta) genes are shown. Unchanged genes are

475 shown in grey and black. For all quantifications, cross SC sections $450 \mu \mathrm{m}$ rostral to the lesion

476 site were quantified. ${ }^{* *} \mathrm{P}<0.001 ;{ }^{* *} \mathrm{P}<0.01$. Scale bars, $50 \mu \mathrm{m}$. 
A Cell proliferation in mstnb mutants

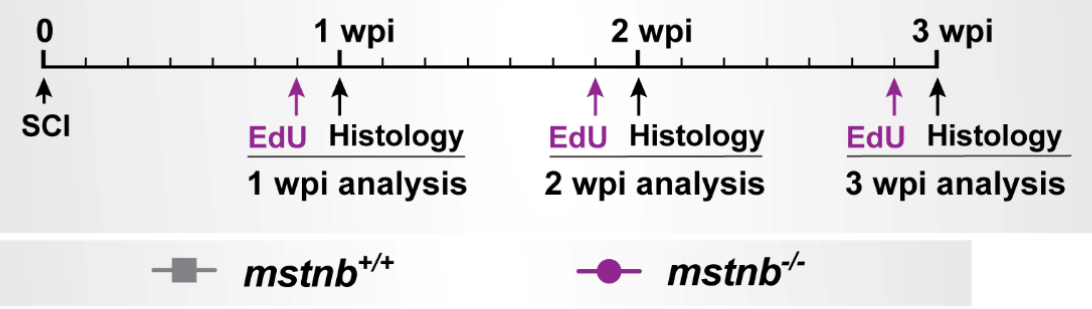

B

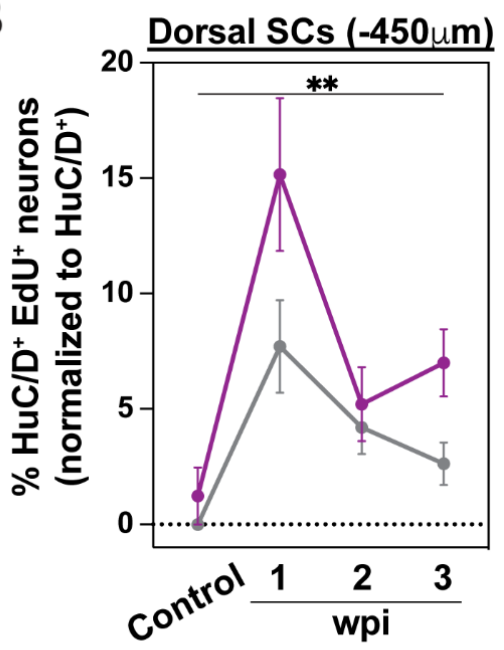

C

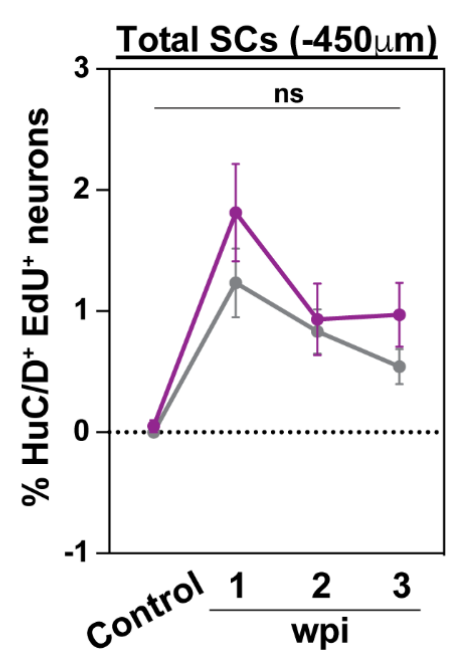

D

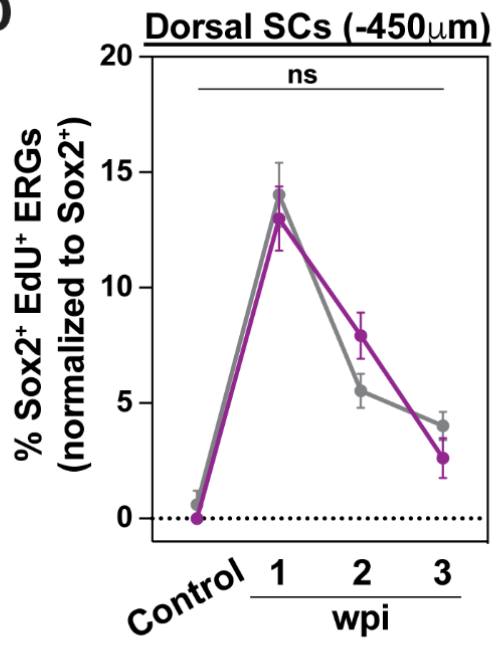

E

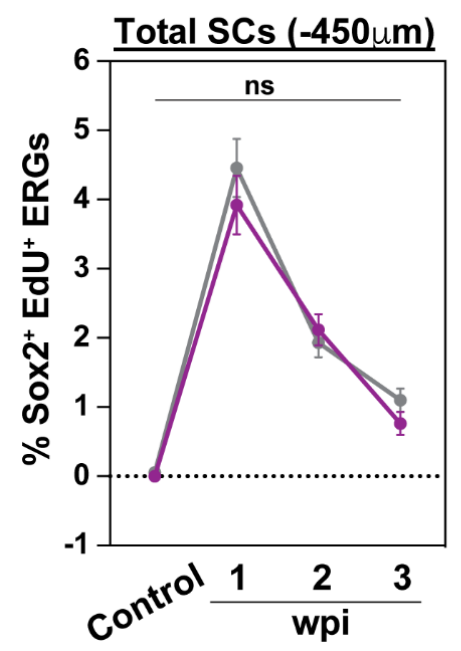


477 Figure S2. Cell proliferation in mstnb mutant zebrafish. (A) Experimental timeline to assess

478 the rates of cell proliferation. $m s t n b^{-/-}$and wild-type siblings were subjected to SC transections.

479 A single EdU injection was performed at either 6, 13, or 20 days post-injury. SC tissues were

480 harvested for analysis at 1, 2, 3 wpi and at 24 hours after EdU injection. Animal numbers are

481 indicated for each genotypes and two independent replicates are shown. (B) Regenerated

$482 \mathrm{HuC} / \mathrm{D}^{+}$EdU ${ }^{+}$neurons were quantified in dorsal SC sections at 1, 2, 3 wpi and uninjured controls.

483 Percent $\mathrm{HuC} / \mathrm{D}^{+} \mathrm{EdU}^{+}$neurons was normalized to the total number of $\mathrm{HuC} / \mathrm{D}^{+}$neurons for each

484 section. (C) Regenerated $\mathrm{HuC} / \mathrm{D}^{+} \mathrm{EdU^{+ }}$ neurons were quantified in total SC sections at 1, 2, 3 wpi

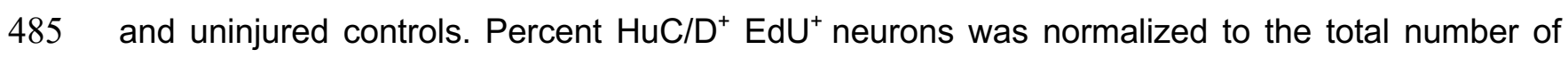

486 nuclei for each section. (D) Sox $2^{+} E d U^{+} E R G s$ were quantified in dorsal SC sections at 1, 2, 3 wpi

487 and uninjured controls. Percent Sox $2^{+} \mathrm{EdU}^{+}$ERGs was normalized to the total number of Sox $2^{+}$

488 ERGs for each section. (E) Sox2 ${ }^{+} E d U^{+}$ERGs were quantified in total SC sections at 1, 2, 3 wpi

489 and uninjured controls. Percent Sox $2^{+} \mathrm{EdU}^{+} \mathrm{ERGs}$ was normalized to the total number of nuclei

490 for each section. For all quantifications, cross SC sections $450 \mu \mathrm{m}$ rostral to the lesion site were

491 quantified. ${ }^{* *} \mathrm{P}<0.01$; ns, not significant. 
Figure S3

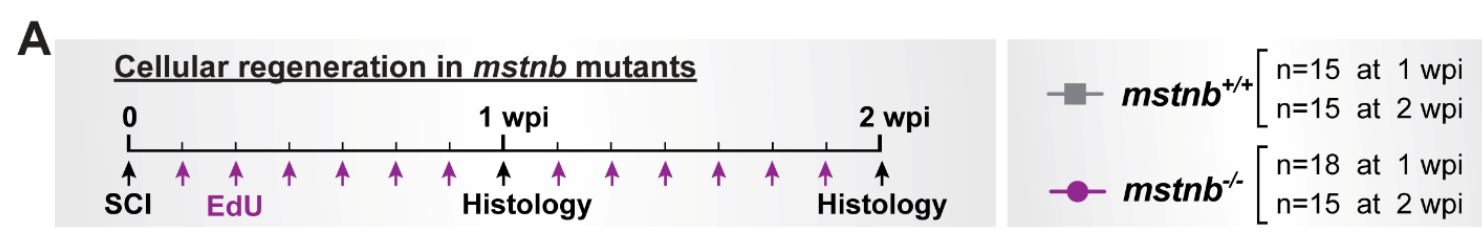

B

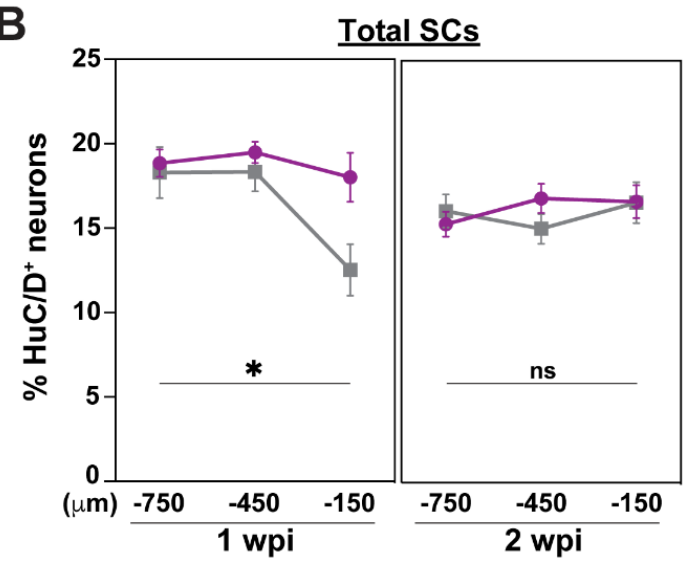

C

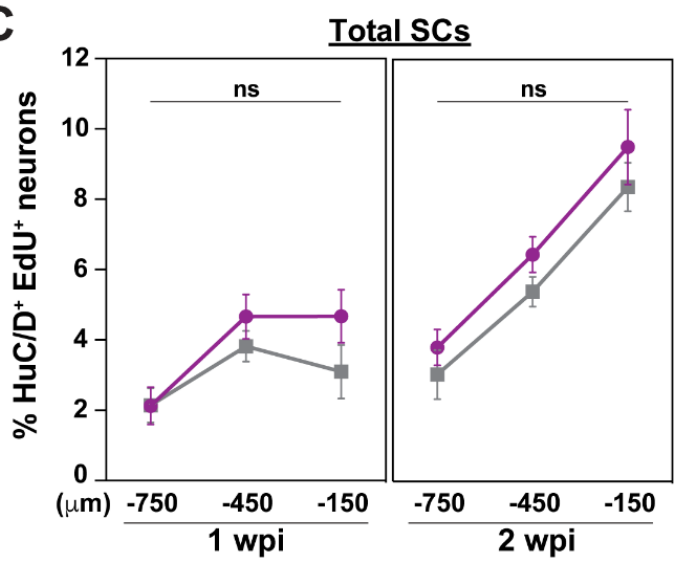


492 Figure S3. Regenerative neurogenesis in mstnb mutant zebrafish. (A) Experimental timeline

493 to assess the rates of neurogenesis and ERG self-renewal. $m s t n b^{-/}$and wild-type siblings were 494 subjected to SC transections and daily EdU injections. SC tissues were harvested for analysis at 4951 or 2 wpi. Animal numbers are indicated for each genotypes and two independent replicates are 496 shown. (C) HuC/D+ neurons were quantified in total SC sections at 1 and 2 wpi. Percent HuC/D 497 neurons was normalized to the total number of nuclei for each section. (D) Regenerated HuC/D+

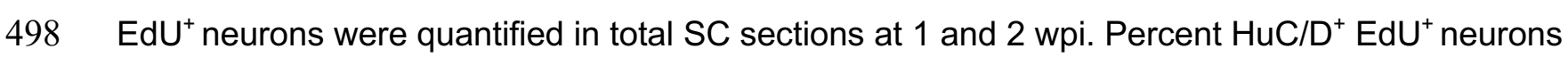
499 was normalized to the total number of nuclei for each section. For all quantifications, cross SC 500 sections at 150,450 , and $750 \mu \mathrm{m}$ rostral to the lesion site were quantified. ${ }^{* *} \mathrm{P}<0.01$; ns, not 501 significant. 


\section{Figure S4}

A

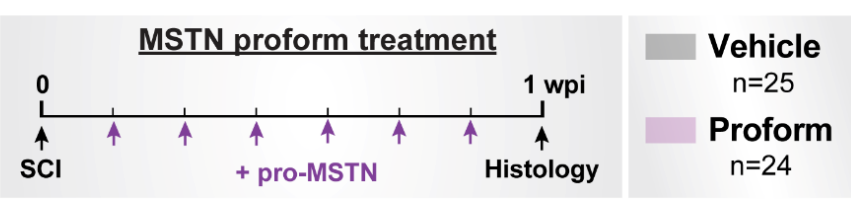

B

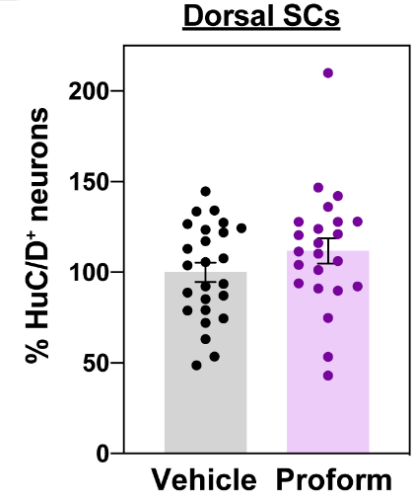

C

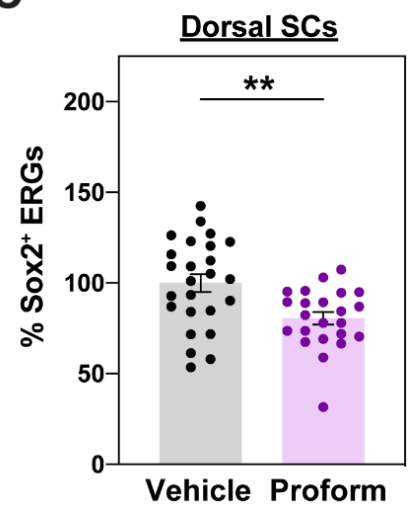


502 Figure S4. Pharmacological Mstn inhibition during SC regeneration. (A) For local Mstnb 503 inhibition, wild-type SCs were subjected to SC transections and daily injections of human 504 recombinant MSTN Proform (pro-MSTN) peptide adjacent to the lesion site. SC tissues were 505 harvested for analysis at 1 wpi. Animal numbers are indicated for each genotypes and two 506 independent replicates are shown. (B) $\mathrm{HuC} / \mathrm{D}^{+}$neurons were quantified in pro-MSTN- and 507 vehicle-treated SCs. Dorsal SC sections at 1 wpi were analyzed. Percent HuC/D ${ }^{+}$neurons was 508 normalized to the total number of nuclei for each section. (C) Sox2 ${ }^{+}$ERGs were quantified in pro509 MSTN- and vehicle-treated SCs. Dorsal SC sections at 1 wpi were analyzed. Percent Sox $2^{+}$ERGs 510 was normalized to the total number of nuclei for each section. For all quantifications, cross SC 511 sections $450 \mu \mathrm{m}$ rostral to the lesion site were quantified. ${ }^{* *} \mathrm{P}<0.01$. 
A

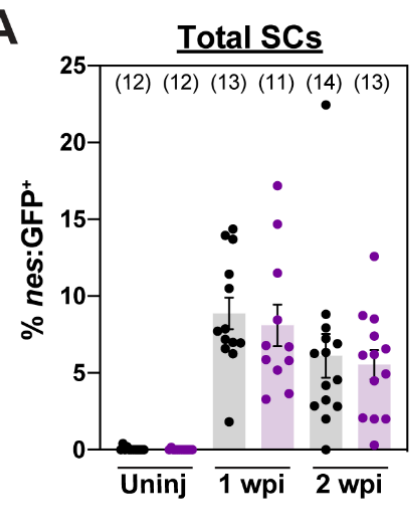

D

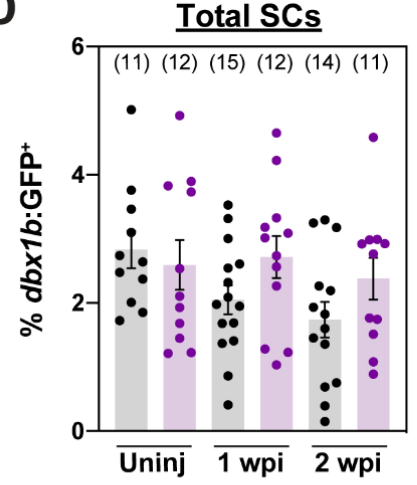

$\mathbf{F}$

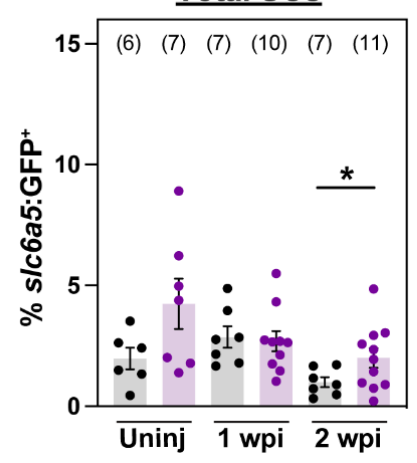

B

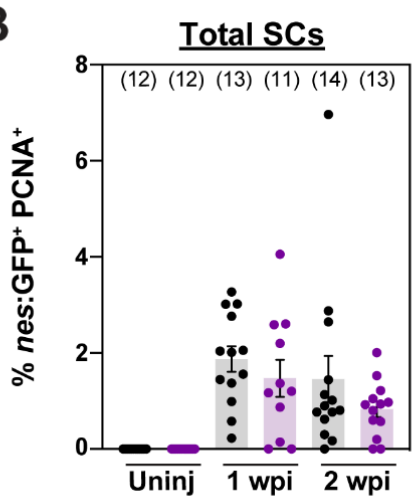

$\mathbf{E}$

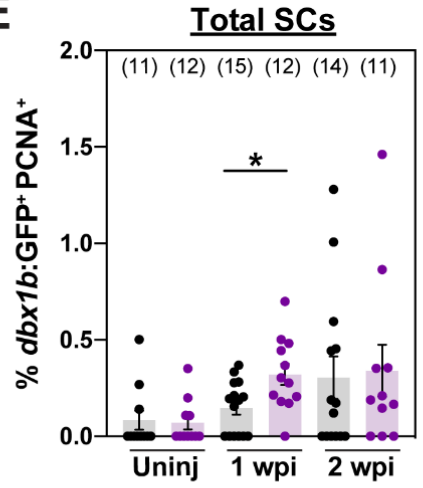

mstnb $^{+/+}$

mstnb $^{-/}$

Figure S5

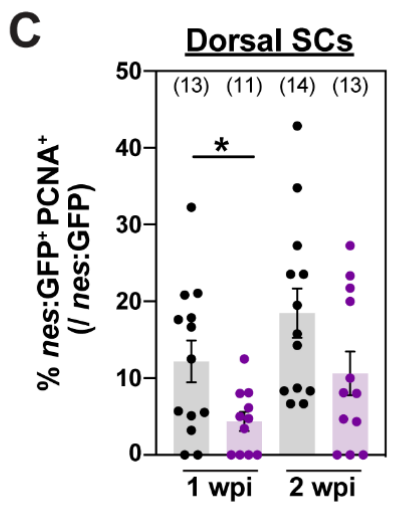


512 Figure S5. Assessment of neuronal progenitors and neurons in mstnb mutant zebrafish.

513 (A) $n e s^{+}$NSCs were quantified in total SC sections. Percent $n e s^{+}$NSCs was normalized to the

514 total number of nuclei for each section. (B) nes $^{+}$PCNA $^{+}$NSCs were quantified in total SC sections.

515 Percent $n e s^{+} \mathrm{PCNA}^{+}$NSCs was normalized to the total number of nuclei for each section. (C)

$516 n$ nes $^{+} \mathrm{PCNA}^{+} \mathrm{NSCs}$ were quantified in dorsal SC sections. Percent nes ${ }^{+} \mathrm{PCNA}^{+} \mathrm{NSCs}$ was

517 normalized to the total number of $n e s^{+}$NSCs for each section. (D) $d b x 1 b^{+}$iNPs were quantified

518 in total SC sections. Percent $d b \times 1 b^{+}$iNPs was normalized to the total number of nuclei for each

519 section. (E) $d b \times 1 b^{+} \mathrm{PCNA}^{+}$iNPs were quantified in total SC sections. Percent $d b \times 1 b^{+} \mathrm{PCNA}^{+}$

520 NSCs was normalized to the total number of nuclei for each section. (F) s/c6a5:GFP ${ }^{+}$glycinergic

521 neurons were quantified in total SC sections. Percent s/c6a5:GFP ${ }^{+}$neurons was normalized to

522 the total number of nuclei for each section. For all quantifications, cross SC sections $450 \mu \mathrm{m}$

523 rostral to the lesion site were quantified. ${ }^{*} \mathrm{P}<0.05$. 


\begin{tabular}{|c|c|c|c|}
\hline Gene & Primer Name & Sequence & Product size \\
\hline \multirow{2}{*}{ birc5b } & bric5b_exon1_fwd & CGAGAAGATTGCCAGTGCACAC & \multirow{2}{*}{70} \\
\hline & bric5b_exon2_rev & CATTCTCACTGGGACAGTGAAC & \\
\hline \multirow{2}{*}{ eloal } & eloal_exon1_fwd & GCTGAAGGAATGTAATGATG & \multirow{2}{*}{74} \\
\hline & eloal_exon2_rev & CAAGTGTGATGTCCAATACTTC & \\
\hline \multirow{2}{*}{ elavl2 } & elavl2_exon3_fwd & GAGATCGAGTCCTGCAAACTCG & \multirow{2}{*}{79} \\
\hline & elavl2_exon4_rev & GCTCCATATAGTTCACAAAGCCG & \\
\hline \multirow{2}{*}{ htr2aa } & htr2aa_exon1_fwd & GTCATGCCAGTCTCCATGGTG & \multirow{2}{*}{74} \\
\hline & htr2aa_exon2_rev & CACATGGGACACAGTGATGC & \\
\hline \multirow{2}{*}{ pou5f3 } & pou5f3_exon3_fwd & GAACGAGGCCGAAAACTCCGAG & \multirow{2}{*}{67} \\
\hline & pou5f3_exon4_rev & CGTGTCGACAAACACCCGTTC & \\
\hline \multirow{2}{*}{ uncx4.1 } & uncx4.1_exon2_fwd & GCTACGTCTAGACCTTGTTGAG & \multirow{2}{*}{65} \\
\hline & uncx4.1_exon3_rev & GCCATTTGGCTCGCCGGTTTTG & \\
\hline \multirow{2}{*}{ znhit3 } & znhit3_exon3_fwd & CAGATCCAGCCTCCAGCAAAC & \multirow{2}{*}{75} \\
\hline & znhit3_exon4_rev & CATCCAGCAGATCCTCAACAGTC & \\
\hline \multirow{2}{*}{ grip2a } & grip2a_exon4_fwd & GCCATCCGCTTTATAGAGCCTG & \multirow{2}{*}{72} \\
\hline & grip2a_exon5_rev & GAGAATTCTGTCGCCAACTTG & \\
\hline \multirow{2}{*}{ slbp } & slbp_exon1_fwd & CGATTACAAATCTAGTGAAGACAG & \multirow{2}{*}{84} \\
\hline & slbp_exon2_rev & CTCAGAATACCATCTGCTCCAC & \\
\hline \multirow{2}{*}{ tph1a } & tph1a_exon6_fwd2 & GAAGACAACATCCCTCAGCTG & \multirow{2}{*}{77} \\
\hline & tph1a_exon7_rev2 & GCCACAGGCCTGATGGTGAAG & \\
\hline \multirow{2}{*}{ ntrk2b } & ntrk2b_exon6_fwd & CTTAAATTCCAGCGAACACCC & \multirow{2}{*}{83} \\
\hline & ntrk2b_exon7_rev & CATTTAGAGTCAGCACCGACTGC & \\
\hline \multirow{2}{*}{$f g f 1 b$} & fgf1b_exon1_fwd & GCAATGGACAAATGTGGAAG & \multirow{2}{*}{76} \\
\hline & fgf1b_exon2_rev & ССТССАТСТTСТСАATGAAGAAAC & \\
\hline
\end{tabular}

524 Table S1. Primer sequences for qRT-PCR. Gene names, primer names, sequences, and 525 product sizes are indicated. 


\section{METHODS}

526 Zebrafish. Adult zebrafish of the Ekkwill, Tubingen, and AB strains were maintained at the

527 Washington University Zebrafish Core Facility. All animal experiments were performed in

528 compliance with institutional animal protocols. Male and female animals between 3 and 9 months

529 of $\sim 2 \mathrm{~cm}$ in length were used. Experimental fish and control siblings of similar size and equal sex

530 distribution were used for all experiments. SC transection surgeries and regeneration analyses

531 were performed in a blinded manner, and 2 to 4 independent experiments were repeated using

532 different clutches of animals. The following previously published zebrafish strains were used:

$533 m s t n^{\text {bns5 }}$ (Dogra et al., 2017), Tg(nes:GFP) (Lam et al., 2009), Tg(dbx1b:GFP) (Satou et al., 2012),

$534 \mathrm{Tg}($ slc6a5:GFP) (McLean et al., 2007).

535 SC transection and treatment. Zebrafish were anaesthetized using MS-222. Fine scissors were 536 used to make a small incision that transects the SC $4 \mathrm{~mm}$ caudal to the brainstem region.

537 Complete transection was visually confirmed at the time of surgery. Injured animals were also 538 assessed at 2 or 3 dpi to confirm loss of swim capacity post-surgery. For sham injuries, animals 539 were anaesthetized, and fine scissors were used to transect skin and muscle tissues without 540 inducing SCl.

541 For pro-MSTN treatment, lyophilized human MSTN proform peptide (BioVision, 4623P-10) was 542 reconstituted in $\mathrm{ddH}_{2} \mathrm{O}$ to a concentration $100 \mathrm{ng} / \mu \mathrm{l}$. Zebrafish were anaesthetized using MS-222. $5432 \mu \mathrm{l}(200 \mathrm{ng})$ of reconstituted peptides were injected daily adjacent and lateral to the SC lesion 544 site. $2 \mu \mathrm{l}$ of $\mathrm{ddH}_{2} \mathrm{O}$ was injected for vehicle controls.

545 For FGF1 treatment, lyophilized human FGF1 protein (PeproTech, 100-17A) was reconstituted in 546 heparin to a concentration $250 \mathrm{ng} / \mu \mathrm{l}$. Sterile Gelfoam Absorbable Gelatin Sponge (Pfizer, 09547 0315-08) was cut into $2 \mathrm{~mm}^{3}$ pieces, soaked with $2 \mu \mathrm{l}$ of recombinant FGF1, then cut into 10 548 smaller pieces (50 ng per piece). Vehicle gelfoam pieces were soaked with $2 \mu$ of heparin 549 solution. At $5 \mathrm{dpi}$, zebrafish were anaesthetized using MS-222 and longitudinal incision lateral 550 and parallel to the SC was made with fine scissors. Injured SC tissues were exposed without 551 causing secondary injuries and gelfoam sponges were places adjacent to the lesion site. The 552 incision was closed and glued using Vetbond tissue adhesive material as previously described 553 (Mokalled et al., 2016). 
554 Bulk RNA sequencing. Two mm SC sections, including the lesion site plus additional rostral and

555 caudal tissue proximal to the lesion, were collected from mstnb mutants and wild-type siblings at

5561 wpi. Uninjured mstnb mutants and wild-type SCs were also collected. Total RNA was prepared

557 using NucleoSpin RNA Plus XS (Clontech, cat\# 740990) and sent for bulk RNA sequencing.

558 TruSeq libraries were prepared and sequenced on Illumina HiSeq 3000 using 50 bp single-end

559 reading strategy. Quality QC and trimming of adapters and short sequences were performed

560 using Fastx. Sequencing reads were mapped to the zebrafish genome (Zv11) using Bowtie2, then

561 assembled and quantified using the Cufflinks and Cuffdiff algorithms. Genes with $\log _{2}$ (fold

562 enrichment) between -1 and 1 or adjusted $p$-value $\geq 0.01$ were considered insignificant. RNA

563 sequencing was performed at the Genome Technology Access Center at Washington University.

564 Analysis was performed in the Bioinformatics Core at the Center for Regenerative Medicine at

565 Washington University.

566 RNA-seq data (GEO accession number : GSE77025) was used to evaluate the expression of Tgf-

$567 \beta$ ligands after complete SC transection. $\log _{2}$ (fold change) is expressed for SCs at 1 wpi relative

568 to the sham injured SCs (Mokalled et al., 2016).

569 Histology. $16 \mu \mathrm{m} \mu \mathrm{m}$ cross cryosections of paraformaldehyde-fixed SC tissue were used. Tissue 570 sections were imaged using a Zeiss AxioVision compound microscope for in situ hybridization or 571 a Zeiss LSM 800 confocal microscope for immunofluorescence. In situ hybridization for mstnb 572 was performed as previously described (Mokalled et al., 2016).

573 For immunohistochemistry, tissue sections were rehydrated in PBT (0.1\% Tween-20 in PBS), 574 then treated with blocking agent ( $5 \%$ goat serum in PBT) for $1 \mathrm{hr}$ at room temperature. For nuclear 575 antigens, sections were treated with $0.2 \%$ TritonX-100 in PBT for 5 minutes and washed 576 thoroughly in PBT prior to the blocking step. Sections were incubated overnight with the indicated 577 primary antibodies, washed in PBT, and treated for $1 \mathrm{hr}$ with secondary antibodies. Following 578 washes, sections were incubated in $1 \mathrm{mg} / \mathrm{mL}$ of Hoechst and mounted in Fluoromount-G 579 mounting media. Primary antibodies used in this study were: rabbit anti-Smad3(S423/425) 580 (Abcam, ab52903, 1:50), rabbit anti-PCNA (GeneTex, GTX124496, 1:500), mouse anti-HuC/D 581 (Invitrogen, A21271, 1:500), mouse anti-Gfap (ZIRC, Zrf1, 1:1000), mouse anti-acetylated $\alpha$ 582 tubulin (Sigma, T6793, 1:1000), chicken anti-GFP (Aves Labs, 1020, 1:1000), rabbit anti-Sox2 583 (GeneTex, 124477, 1:250). Secondary antibodies (Invitrogen, 1:200) used in this study were 
584 Alexa Fluor 488- or Alexa Fluor 594- conjugated goat anti-rabbit, anti-mouse, or anti-chicken 585 antibodies.

586 For simultaneous labeling with rabbit anti-Sox2 (GeneTex, 124477, 1:250) and rabbit anti587 pSmad3(S423/425) (abcam, ab52903, 1:50) (Fig. 1B), unconjugated Fab Fragment Goat Anti588 Rabbit IgG(H+L) (Jackson ImmunoResearch, 111-007-003) and donkey anti-goat 568 (Thermo 589 fisher: A-11057) antibodies were used for pSmad3 labeling. Sox2 was labeled using donkey anti590 rabbit-488 (Jackson ImmunoResearch, 711-547-003).

591 EdU Staining was adapted from a a previously described protocol (Salic and Mitchison, 2008). 592 Briefly, zebrafish were anaesthetized using MS-222 and subjected to intraperitoneal EdU 593 injections. $12.5 \mathrm{mM}$ EdU (Sigma 900584) diluted in PBS was used. A single injection (Fig. 3 and 594 S2) or multiple, daily injections (Fig. 4 and S3) were performed and paraformaldehyde-fixed cryosections were used. Sections were rehydrated in PBT for 10 min then incubated with freshly prepared staining solution for $30 \mathrm{~min}$ (100 mM Tris (Sigma, T6066) pH 8.5; $1 \mathrm{mM} \mathrm{CuSO}_{4}$ (Sigma, C1297); $10 \mu \mathrm{M}$ fluorescent azide; and $100 \mathrm{mM}$ ascorbic acid (Sigma, A5960)).

Cell counting. Cell counting was performed using a customized Fiji script (adapting ITCN: Image based Tool for counting nuclei- https://imagej.nih.gov/ij/plugins/itcn.html). Orthogonal projections

600 of individual image stacks were generated using Zen software. A Customized Fiji script 601 incorporated user-defined inputs to define channels (including Hoechst), to determine the center 602 of the central canal, and to outline SC perimeters. SC tissues dorsal to the central canal center 603 was considered "dorsal SC". SC tissues ventral to the central canal center was considered 604 "ventral SC". To quantify nuclei, the following parameters were set in ITCN counter: width, 15; 605 minimal distance, 7.5; threshold, 0.4. For each staining, thresholds were user-defined. Raw 606 counts from Fiji were processed using a customized R Studio script.

607 Swim endurance assays. Zebrafish were exercised in groups of 8-12 in a $5 \mathrm{~L}$ swim tunnel device 608 (Loligo, cat\# SW100605L, 120V/60Hz). After 10 minutes of acclimation inside the enclosed 609 tunnel, water current velocity was increased every two minutes and fish swam against the current 610 until they reached exhaustion. Exhausted animals were removed from the chamber without 611 disturbing the remaining fish. Swim time and current velocity at exhaustion were recorded. Results 612 were expressed as means \pm SEM. An unpaired two-tailed Student's t-test with Welch correction 
613 was performed using the Prism software to determine statistical significance of swim times 614 between groups.

615 Swim behavior assays. Zebrafish were divided into groups of 5 in a $5 \mathrm{~L}$ swim tunnel device 616 (Loligo, cat\# SW100605L, 120V/60Hz). Each group was allowed to swim for a total of $15 \mathrm{~min}$ 617 under zero to low current velocities ( $5 \mathrm{~min}$ at $0 \mathrm{~cm} / \mathrm{s}, 5 \mathrm{~min}$ at $10 \mathrm{~cm} / \mathrm{s}$, and $5 \mathrm{~min}$ at $15 \mathrm{~cm} / \mathrm{s}$ ). The 618 entire swim behavior was recorded using high-speed camera (iDS, USB 3.0 color video camera) 619 with following settings: aspect ratio, 1:4; pixel clock, 344; frame rate, 70 frames/s; exposure time: $620 \quad 0.29$; aperture, 1.4 to 2; maximum frames; 63,000. Movies were converted to 20 frames/s and 621 analyzed using a customized Fiji macro. For each frame, animals/objects $>1500 \mathrm{px}^{2}$ were 622 identified, and the $X Y$ coordinates were derived for each animal/object. Frame were 623 independently, and animal/object tracking was completed using a customized R Studio script. The 624 script aligned coordinates, calculated swim metrics considering three separate frame windows 625 (Frames 0-6000 at $0 \mathrm{~cm} / \mathrm{s}$; frames $6001-12000$ at $10 \mathrm{~cm} / \mathrm{s}$, and frames $12001-18001$ at $20 \mathrm{~cm} / \mathrm{s}$ ).

Glial bridging. GFAP immunohistochemistry was performed on serial transverse sections. The cross-sectional area of the glial bridge and the area of the intact SC rostral to the lesion were measured using ImageJ software. Bridging was calculated as a ratio of these measurements. Mann Whitney tests were performed using Prism software to determine statistical significance between groups.

631 Axon tracing. Anterograde axon tracing was performed on adult fish at 4 wpi. Fish were 632 anaesthetized using MS-222 and fine scissors were used to transect the cord $4 \mathrm{~mm}$ rostral to the 633 lesion site. Biocytin-soaked Gelfoam Gelatin Sponge was applied at the new injury site (Gelfoam, 634 Pfizer, cat\# 09-0315-08; Biocytin, saturated solution, Sigma, cat\# B4261). Fish were euthanized 6356 hours post-treatment and Biocytin was histologically detected using Alexa Fluor 594-conjugated 636 Streptavidin (Molecular Probes, cat\# S-11227). Biocytin-labeled axons were quantified using the 637 "threshold" and "particle analysis" tools in the Fiji software. Four sections per fish at 0.5 (proximal) 638 and 2 (distal) $\mathrm{mm}$ caudal to the lesion core, and 2 sections $1 \mathrm{~mm}$ rostral to the lesion, were 639 analyzed. Axon growth was normalized to the efficiency of Biocytin labeling rostral to the lesion 640 for each fish. The axon growth index was then normalized to the control group for each 641 experiment. Mann-Whitney tests were performed using Prism software to determine statistical 642 significance between groups 
643 Quantitative real time PCR. Two mm SC sections, including the lesion site plus additional rostral 644 and caudal tissue proximal to the lesion, were collected for qRT-PCR. Total RNA was prepared 645 using NucleoSpin RNA Plus XS (Clontech, cat\# 740990) and cDNA was synthesized using the 646 Maxima First Strand cDNA Synthesis Kit (ThermoFisher, cat\# K1672) according to manufacturer's 647 specifications. Quantitative PCR was completed using the Luna polymerase master mix (NEB, 648 cat\# M3003) using gene-specific primers (Table S1). Primers were designed to flank introns and 649 were confirmed to not amplify project from genomic DNA. To determine primer efficiency, a 650 standard curve was generated for each primer set using cDNA pooled from wild-type embryos at 651 1, 3, and 5 days post-fertilization. qRT-PCR was performed on a Bio-Rad CFX Connect Real652 Time System. For each gene, $\log _{2}$ (fold change) was calculated using the DCq method and 653 normalized to eif1a as a loading control and to control gene expression for each experiment. 


\section{REFERENCES}

Adolf, B., Chapouton, P., Lam, C., Topp, S., Tannhäuser, B., Strähle, U., Götz, M., and BallyCuif, L. (2006). Conserved and acquired features of adult neurogenesis in the zebrafish telencephalon. Developmental biology 295.

Alizadeh, A., Dyck, S., and Karimi-Abdolrezaee, S. (2019). Traumatic Spinal Cord Injury: An Overview of Pathophysiology, Models and Acute Injury Mechanisms. Frontiers in neurology 10.

Bagheri-Mohammadi, S. (2021). Adult neurogenesis and the molecular signalling pathways in brain: the role of stem cells in adult hippocampal neurogenesis. Int J Neurosci, 1-13.

Barbosa, J., Sanchez-Gonzalez, R., Di Giaimo, R., Baumgart, E., Theis, F., Götz, M., and Ninkovic, J. (2015). Neurodevelopment. Live imaging of adult neural stem cell behavior in the intact and injured zebrafish brain. Science (New York, NY) 348.

Barnabe-Heider, F., Goritz, C., Sabelstrom, H., Takebayashi, H., Pfrieger, F.W., Meletis, K., and Frisen, J. (2010). Origin of new glial cells in intact and injured adult spinal cord. Cell Stem Cell 7, 470-482.

Barreiro-Iglesias, A., Mysiak, K.S., Scott, A.L., Reimer, M.M., Yang, Y., Becker, C.G., and Becker, T. (2015). Serotonin Promotes Development and Regeneration of Spinal Motor Neurons in Zebrafish. Cell Rep 13, 924-932.

Becker, T., Bernhardt, R., Reinhard, E., Wullimann, M., Tongiorg, i.E., and Schachner, M. (1998). Readiness of zebrafish brain neurons to regenerate a spinal axon correlates with differential expression of specific cell recognition molecules. The Journal of neuroscience : the official journal of the Society for Neuroscience 18 .

Becker, T., Wullimann, M., Becker, C., Bernhardt, R., and Schachner, M. (1997). Axonal regrowth after spinal cord transection in adult zebrafish. The Journal of comparative neurology 377.

Chapouton, P., Skupien, P., Hesl, B., Coolen, M., Moore, J., Madelaine, R., Kremmer, E., FausKessler, T., Blader, P., Lawson, N., et al. (2010). Notch activity levels control the balance between quiescence and recruitment of adult neural stem cells. The Journal of neuroscience : the official journal of the Society for Neuroscience 30 .

Dogra, D., Ahuja, S., Kim, H.T., Rasouli, S.J., Stainier, D.Y.R., and Reischauer, S. (2017). Opposite effects of Activin type 2 receptor ligands on cardiomyocyte proliferation during development and repair. Nat Commun 8, 1902.

Goldshmit, Y., Sztal, T.E., Jusuf, P.R., Hall, T.E., Nguyen-Chi, M., and Currie, P.D. (2012). Fgfdependent glial cell bridges facilitate spinal cord regeneration in zebrafish. J Neurosci 32, 7477 7492 . 
Grandel, H., Kaslin, J., Ganz, J., Wenzel, I., and Brand, M. (2006). Neural stem cells and neurogenesis in the adult zebrafish brain: origin, proliferation dynamics, migration and cell fate. Developmental biology 295.

Hachem, L., Ahuja, C., and Fehlings, M. (2017). Assessment and management of acute spinal cord injury: From point of injury to rehabilitation. The journal of spinal cord medicine 40 .

Hinckley, C., Seebach, B., and Ziskind-Conhaim, L. (2005). Distinct roles of glycinergic and GABAergic inhibition in coordinating locomotor-like rhythms in the neonatal mouse spinal cord. Neuroscience 131 .

Horky, L.L., Galimi, F., Gage, F.H., and Horner, P.J. (2006). Fate of endogenous stem/progenitor cells following spinal cord injury. J Comp Neurol 498, 525-538.

Horner, P.J., Power, A.E., Kempermann, G., Kuhn, H.G., Palmer, T.D., Winkler, J., Thal, L.J., and Gage, F.H. (2000). Proliferation and differentiation of progenitor cells throughout the intact adult rat spinal cord. J Neurosci 20, 2218-2228.

Hsu, Y., Lee, D., Chen, S., Liao, W., Lin, J., Chiu, W., and Chiu, I. (2009). Brain-specific 1B promoter of FGF1 gene facilitates the isolation of neural stem/progenitor cells with self-renewal and multipotent capacities. Developmental dynamics : an official publication of the American Association of Anatomists 238.

Jovanović, K., Petrov, T., and Stein, R. (1999). Effects of inhibitory neurotransmitters on the mudpuppy (Necturus maculatus) locomotor pattern in vitro. Experimental brain research 129.

Kambadur, R., Sharma, M., Smith, T.P., and Bass, J.J. (1997). Mutations in myostatin (GDF8) in double-muscled Belgian Blue and Piedmontese cattle. Genome Res 7, 910-916.

Keatinge, M., Tsarouchas, T.M., Munir, T., Porter, N.J., Larraz, J., Gianni, D., Tsai, H.H., Becker, C.G., Lyons, D.A., and Becker, T. (2021). CRISPR gRNA phenotypic screening in zebrafish reveals pro-regenerative genes in spinal cord injury. PLoS Genet 17, e1009515.

Kerrison, J.B., Lewis, R.N., Otteson, D.C., and Zack, D.J. (2005). Bone morphogenetic proteins promote neurite outgrowth in retinal ganglion cells. Mol Vis 11, 208-215.

Klatt Shaw, D., and Mokalled, M.H. (2021). Efficient CRISPR/Cas9 mutagenesis for neurobehavioral screening in adult zebrafish. G3 (Bethesda).

Klatt Shaw, D., Saraswathy, V.M., Zhou, L., McAdow, A.R., Burris, B., Butka, E., Morris, S.A., Dietmann, S., and Mokalled, M.H. (2021). Localized EMT reprograms glial progenitors to promote spinal cord repair. Dev Cell 56, 613-626 e617.

Kroehne, V., Freudenreich, D., Hans, S., Kaslin, J., and Brand, M. (2011). Regeneration of the adult zebrafish brain from neurogenic radial glia-type progenitors. Development (Cambridge, England) 138. 
Kuscha, V., Frazer, S., Dias, T., Hibi, M., Becker, T., and Becker, C. (2012a). Lesion-induced generation of interneuron cell types in specific dorsoventral domains in the spinal cord of adult zebrafish. The Journal of comparative neurology 520.

Kuscha, V., Frazer, S.L., Dias, T.B., Hibi, M., Becker, T., and Becker, C.G. (2012b). Lesioninduced generation of interneuron cell types in specific dorsoventral domains in the spinal cord of adult zebrafish. J Comp Neurol 520, 3604-3616.

Lam, C., März, M., and Strähle, U. (2009). gfap and nestin reporter lines reveal characteristics of neural progenitors in the adult zebrafish brain. Developmental dynamics : an official publication of the American Association of Anatomists 238.

Langley, B., Thomas, M., Bishop, A., Sharma, M., Gilmour, S., and Kambadur, R. (2002). Myostatin inhibits myoblast differentiation by down-regulating MyoD expression. J Biol Chem 277, 49831-49840.

Le, W., and Yao, J. (2017). The Effect of Myostatin (GDF-8) on Proliferation and Tenocyte Differentiation of Rat Bone Marrow-Derived Mesenchymal Stem Cells. The journal of hand surgery Asian-Pacific volume 22.

Li, L., and Clevers, H. (2010). Coexistence of quiescent and active adult stem cells in mammals. Science $327,542-545$.

Li, S., Gu, X., and Yi, S. (2017). The Regulatory Effects of Transforming Growth Factor- $\beta$ on Nerve Regeneration. Cell transplantation 26.

Lim, S., McMahon, C., Matthews, K., Devlin, G., Elston, M., and Conaglen, J. (2018). Absence of Myostatin Improves Cardiac Function Following Myocardial Infarction. Heart, lung \& circulation 27.

Magga, J., Vainio, L., Kilpio, T., Hulmi, J.J., Taponen, S., Lin, R., Rasanen, M., Szabo, Z., Gao, E., Rahtu-Korpela, L., et al. (2019). Systemic Blockade of ACVR2B Ligands Protects Myocardium from Acute Ischemia-Reperfusion Injury. Mol Ther 27, 600-610.

März, M., Chapouton, P., Diotel, N., Vaillant, C., Hesl, B., Takamiya, M., Lam, C., Kah, O., BallyCuif, L., and Strähle, U. (2010). Heterogeneity in progenitor cell subtypes in the ventricular zone of the zebrafish adult telencephalon. Glia 58.

Massagué, J. (2012). TGF $\beta$ signalling in context. Nature reviews Molecular cell biology 13.

McCroskery, S., Thomas, M., Maxwell, L., Sharma, M., and Kambadur, R. (2003). Myostatin negatively regulates satellite cell activation and self-renewal. J Cell Biol 162, 1135-1147.

McCroskery, S., Thomas, M., Platt, L., Hennebry, A., Nishimura, T., McLeay, L., Sharma, M., and Kambadur, R. (2005). Improved muscle healing through enhanced regeneration and reduced fibrosis in myostatin-null mice. J Cell Sci 118, 3531-3541. 
McLean, D., Fan, J., Higashijima, S., Hale, M., and Fetcho, J. (2007). A topographic map of recruitment in spinal cord. Nature 446.

Meletis, K., Barnabe-Heider, F., Carlen, M., Evergren, E., Tomilin, N., Shupliakov, O., and Frisen, J. (2008). Spinal cord injury reveals multilineage differentiation of ependymal cells. PLoS Biol 6 , e182.

Mokalled, M.H., Patra, C., Dickson, A.L., Endo, T., Stainier, D.Y., and Poss, K.D. (2016). Injuryinduced ctgfa directs glial bridging and spinal cord regeneration in zebrafish. Science 354, 630634.

Muthusamy, N., Brumm, A., Zhang, X., Carmichael, S.T., and Ghashghaei, H.T. (2018). Foxj1 expressing ependymal cells do not contribute new cells to sites of injury or stroke in the mouse forebrain. Sci Rep 8, 1766.

Ogai, K., Nakatani, K., Hisano, S., Sugitani, K., Koriyama, Y., and Kato, S. (2014). Function of Sox2 in ependymal cells of lesioned spinal cords in adult zebrafish. Neuroscience research 88 .

Oyinbo, C. (2011). Secondary injury mechanisms in traumatic spinal cord injury: a nugget of this multiply cascade. Acta neurobiologiae experimentalis 71 .

Pierani, A., Moran-Rivard, L., Sunshine, M., Littman, D., Goulding, M., and Jessell, T. (2001). Control of interneuron fate in the developing spinal cord by the progenitor homeodomain protein Dbx1. Neuron 29.

Reimer, M., Kuscha, V., Wyatt, C., Sörensen, I., Frank, R., Knüwer, M., Becker, T., and Becker, C. (2009). Sonic hedgehog is a polarized signal for motor neuron regeneration in adult zebrafish. The Journal of neuroscience : the official journal of the Society for Neuroscience 29.

Reimer, M.M., Norris, A., Ohnmacht, J., Patani, R., Zhong, Z., Dias, T.B., Kuscha, V., Scott, A.L., Chen, Y.C., Rozov, S., et al. (2013). Dopamine from the brain promotes spinal motor neuron generation during development and adult regeneration. Dev Cell 25, 478-491.

Reimer, M.M., Sorensen, I., Kuscha, V., Frank, R.E., Liu, C., Becker, C.G., and Becker, T. (2008). Motor neuron regeneration in adult zebrafish. The Journal of neuroscience : the official journal of the Society for Neuroscience $28,8510-8516$.

Ren, Y., Ao, Y., O'Shea, T.M., Burda, J.E., Bernstein, A.M., Brumm, A.J., Muthusamy, N., Ghashghaei, H.T., Carmichael, S.T., Cheng, L., et al. (2017). Ependymal cell contribution to scar formation after spinal cord injury is minimal, local and dependent on direct ependymal injury. Sci Rep 7, 41122.

Rodgers, B.D., Wiedeback, B.D., Hoversten, K.E., Jackson, M.F., Walker, R.G., and Thompson, T.B. (2014). Myostatin stimulates, not inihibits, C2C12 myoblast proliferation. Endocrinology 155, 670-675. 
Rothenaigner, I., Krecsmarik, M., Hayes, J., Bahn, B., Lepier, A., Fortin, G., Götz, M., Jagasia, R., and Bally-Cuif, L. (2011). Clonal analysis by distinct viral vectors identifies bona fide neural stem cells in the adult zebrafish telencephalon and characterizes their division properties and fate. Development (Cambridge, England) 138.

Salic, A., and Mitchison, T. (2008). A chemical method for fast and sensitive detection of DNA synthesis in vivo. Proceedings of the National Academy of Sciences of the United States of

794 America 105.

795

796

797

798

799

800

801

802

803

804

805

806

807

808

809

810

811

812

813

814

815

816

817

818

819

Sartori, R., Gregorevic, P., and Sandri, M. (2014). TGF $\beta$ and BMP signaling in skeletal muscle: potential significance for muscle-related disease. Trends in endocrinology and metabolism: TEM 25 .

Satou, C., Kimura, Y., and Higashijima, S. (2012). Generation of multiple classes of V0 neurons in zebrafish spinal cord: progenitor heterogeneity and temporal control of neuronal diversity. The Journal of neuroscience : the official journal of the Society for Neuroscience 32.

Schuelke, M., Wagner, K.R., Stolz, L.E., Hubner, C., Riebel, T., Komen, W., Braun, T., Tobin, J.F., and Lee, S.J. (2004). Myostatin mutation associated with gross muscle hypertrophy in a child. N Engl J Med 350, 2682-2688.

Shah, P.T., Stratton, J.A., Stykel, M.G., Abbasi, S., Sharma, S., Mayr, K.A., Koblinger, K., Whelan, P.J., and Biernaskie, J. (2018). Single-Cell Transcriptomics and Fate Mapping of Ependymal Cells Reveals an Absence of Neural Stem Cell Function. Cell 173, 1045-1057 e1049.

Sharma, M., McFarlane, C., Kambadur, R., Kukreti, H., Bonala, S., and Srinivasan, S. (2015). Myostatin: expanding horizons. IUBMB life 67.

Sibilla, S., and Ballerini, L. (2009). GABAergic and glycinergic interneuron expression during spinal cord development: dynamic interplay between inhibition and excitation in the control of ventral network outputs. Progress in neurobiology 89.

Silva, N., Sousa, N., Reis, R., and Salgado, A. (2014). From basics to clinical: a comprehensive review on spinal cord injury. Progress in neurobiology 114.

Singh, A., Tetreault, L., Kalsi-Ryan, S., Nouri, A., and Fehlings, M. (2014). Global prevalence and incidence of traumatic spinal cord injury. Clinical epidemiology 6.

Sofroniew, M.V. (2018). Dissecting spinal cord regeneration. Nature 557, 343-350.

Taylor, W.E., Bhasin, S., Artaza, J., Byhower, F., Azam, M., Willard, D.H., Jr., Kull, F.C., Jr., and Gonzalez-Cadavid, N. (2001). Myostatin inhibits cell proliferation and protein synthesis in C2C12 muscle cells. Am J Physiol Endocrinol Metab 280, E221-228. 
Than-Trong, E., Kiani, B., Dray, N., Ortica, S., Simons, B., Rulands, S., Alunni, A., and BallyCuif, L. (2020). Lineage hierarchies and stochasticity ensure the long-term maintenance of adult neural stem cells. Science advances 6 .

Than-Trong, E., Ortica-Gatti, S., Mella, S., Nepal, C., Alunni, A., and Bally-Cuif, L. (2018). Neural stem cell quiescence and stemness are molecularly distinct outputs of the Notch3 signalling cascade in the vertebrate adult brain. Development (Cambridge, England) 145.

Tran, A.P., Warren, P.M., and Silver, J. (2021). New insights into glial scar formation after spinal cord injury. Cell Tissue Res.

Uribe, V., Ramadass, R., Dogra, D., Rasouli, S.J., Gunawan, F., Nakajima, H., Chiba, A., Reischauer, S., Mochizuki, N., and Stainier, D.Y.R. (2018). In vivo analysis of cardiomyocyte proliferation during trabeculation. Development 145.

Wallner, C., Jaurich, H., Wagner, J., Becerikli, M., Harati, K., Dadras, M., Lehnhardt, M., and Behr, B. (2017). Inhibition of GDF8 (Myostatin) accelerates bone regeneration in diabetes mellitus type 2. Scientific reports 7.

Whittemore, L.A., Song, K., Li, X., Aghajanian, J., Davies, M., Girgenrath, S., Hill, J.J., Jalenak, M., Kelley, P., Knight, A., et al. (2003). Inhibition of myostatin in adult mice increases skeletal muscle mass and strength. Biochem Biophys Res Commun 300, 965-971.

Wu, H.H., Ivkovic, S., Murray, R.C., Jaramillo, S., Lyons, K.M., Johnson, J.E., and Calof, A.L. (2003). Autoregulation of neurogenesis by GDF11. Neuron 37, 197-207.

Yamamoto, S., Yamamoto, N., Kitamura, T., Nakamura, K., and Nakafuku, M. (2001). Proliferation of parenchymal neural progenitors in response to injury in the adult rat spinal cord. Exp Neurol 172, 115-127.

Zhu, X., Hadhazy, M., Wehling, M., Tidball, J., and McNally, E. (2000). Dominant negative myostatin produces hypertrophy without hyperplasia in muscle. FEBS letters 474. 